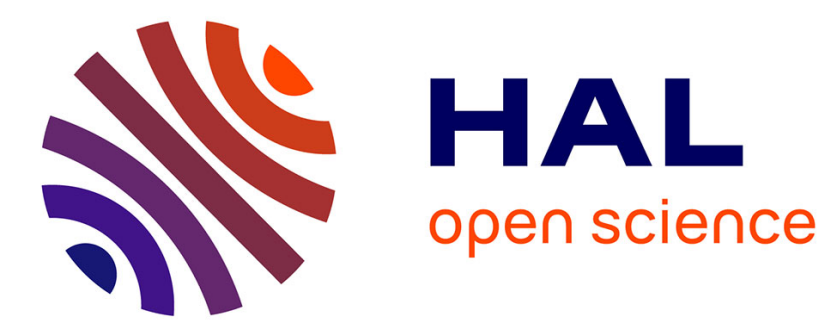

\title{
Size of planar domains and existence of minimizers of the Ginzburg-Landau energy with semi-stiff boundary conditions
}

Petru Mironescu

\section{- To cite this version:}

Petru Mironescu. Size of planar domains and existence of minimizers of the Ginzburg-Landau energy with semi-stiff boundary conditions. Contemp. Math. Fundamental Directions, 2013, 47, pp.78-107. hal-00747657v2

\author{
HAL Id: hal-00747657 \\ https://hal.science/hal-00747657v2
}

Submitted on 31 Dec 2012

HAL is a multi-disciplinary open access archive for the deposit and dissemination of scientific research documents, whether they are published or not. The documents may come from teaching and research institutions in France or abroad, or from public or private research centers.
L'archive ouverte pluridisciplinaire HAL, est destinée au dépôt et à la diffusion de documents scientifiques de niveau recherche, publiés ou non, émanant des établissements d'enseignement et de recherche français ou étrangers, des laboratoires publics ou privés. 


\title{
SIZE OF PLANAR DOMAINS AND EXISTENCE OF MINIMIZERS OF THE GINZBURG-LANDAU ENERGY WITH SEMI-STIFF BOUNDARY CONDITIONS
}

\author{
PETRU MIRONESCU
}

\begin{abstract}
The Ginzburg-Landau energy with semi-stiff boundary conditions is an intermediate model between the full Ginzburg-Landau equations, which make appear both a condensate wave function and a magnetic potential, and the simplified Ginzburg-Landau model, coupling the condensate wave function to a Dirichlet boundary condition. In the semi-stiff model, there is no magnetic potential. The boundary data is not fixed, but circulation is prescribed on the boundary. Mathematically, this leads to prescribing the degrees on the components of the boundary. The corresponding problem is variational, but non compact: in general, energy minimizers do not exist. Existence of minimizers is governed by the topology and the size of the underlying domain. We propose here various notions of domain size related to existence of minimizers, and discuss existence of minimizers or critical points, as well as their uniqueness and asymptotic behavior. We also present the state of the art in the study of this model, accounting results obtained during the last decade by L.V. Berlyand, M. Dos Santos, A. Farina, D. Golovaty, X. Lamy, V. Rybalko, E. Sandier, and the author.
\end{abstract}

\section{Contents}

1. Introduction

2. Winding number of circle-valued maps 3

3. Non compactness $\quad 5$

4. Size of domains $\quad 6$

5. Critical points in multiply connected domains $\quad 15$

6. Critical points in simply connected domains $\quad 17$

7. Asymptotics and uniqueness 21

8. Perspectives and open problems 26

References $\quad 27$

\section{INTRODUCTION}

Two-dimensional ${ }^{1}$ superconductivity is described by the Ginzburg-Landau (GL, in short) energy

$$
\frac{1}{2} \int_{\Omega}|\nabla u-\imath A u|^{2}+\frac{1}{4 \varepsilon^{2}} \int_{\Omega}\left(1-|u|^{2}\right)^{2}+\frac{1}{2} \int_{\Omega}\left|d A-h_{\text {ext }}\right|^{2} .
$$

Here, $\Omega \subset \mathbb{R}^{2}$ is smooth bounded simply connected, $u: \Omega \rightarrow \mathbb{C}$ is the condensate wave function, $A: \Omega \rightarrow \mathbb{R}^{2}$ is the magnetic potential, and $h_{\text {ext }}$ is the external magnetic field. $|u|^{2}$ is a density (density of Cooper pairs of electrons) and a measure of superconductivity: $|u|^{2}$ close to 1 indicates a superconductor state, $|u|$ close to 0 corresponds to the normal state. $\kappa=\frac{1}{\varepsilon}$ is the GL parameter. According to the values of $\varepsilon$ and $h_{\text {ext }}$, the density $|u|^{2}$ of an energy minimizer $(u, A)$ tends to be (locally) close to either 1 or 0 . Typically, either $|u|^{2}$ is uniformly close to 1 (Meissner states) or $|u|^{2}$ is close to 1 most of time, but there are small regions where $|u|^{2}$ is close to 0 . These small regions

1991 Mathematics Subject Classification. 517.9.

Key words: Ginzburg-Landau, capacity, non compact, degree, mountain pass

Subject classification: 35J20, 42B37

${ }^{1}$ As usual in material science, the two-dimensional domain $\Omega$ is a cross section of a three-dimensional cylinder, and the unknown functions are supposed to be independent of the $z$-variable. 
are "vortices", and though there is no universally accepted definition of vortices, they are understood as points or regions where $|u|^{2}$ is, in an appropriate sense, close to 0 . See the monograph [32] of Sandier and Serfaty for a mathematical theory of GL vortices in superconductivity.

The analysis in [32] relies partly on tools previously developed by Bethuel, Brezis and Hélein [14] for the simpler model consisting in minimizing the simplified GL energy $\frac{1}{2} \int_{\Omega}|\nabla u|^{2}+\frac{1}{4 \varepsilon^{2}} \int_{\Omega}(1-$ $\left.|u|^{2}\right)^{2}$. Clearly, if no restriction is imposed on $\Omega$, then the absolute minimizers are constant of modulus 1 . However, if we impose the Dirichlet condition $u=g$ on $\partial \Omega$ and if $g$ forces $u$ to vanish, then the Dirichlet condition provides a vortex creation mechanism. This is the case if, for example, $g: \partial \Omega \rightarrow \mathbb{S}^{1}$ has a non zero winding number (degree), and [14] is devoted to the minimization of the simplified GL functional subject to non zero winding number Dirichlet boundary condition $g$. Though this model is not physically realistic, it captures some of the most important features of the full model, namely vortex formation and the fact that vortices do mutually repel and are repelled far away from the boundary. The same features were proved for the full model when there are only few vortices [32].

In other models, vortices can be attracted by the boundary. For example, if we consider the simplified GL energy with the constraint $|u|=1$ on the boundary, then the only stable critical points in simply connected domains are constants [33]; in a certain sense, all possible vortices are expelled at the boundary.

A mathematically interesting intermediate model, simpler than the full GL model and allowing near boundary vortices, was proposed by Berlyand and Voss [12]. Let $\Omega \subset \mathbb{R}^{2}$ be a smooth bounded domain and let $\Gamma_{0}, \ldots \Gamma_{k}$ denote the components of $\partial \Omega$, with $\Gamma_{0}$ enclosing $\Omega$. Consider a collection of integers $\mathbf{d}=\left(d_{0}, \ldots, d_{k}\right) \in \mathbb{Z}^{k+1}$ and the simplified energy

$$
E_{\varepsilon}(u)=\frac{1}{2} \int_{\Omega}|\nabla u|^{2}+\frac{1}{4 \varepsilon^{2}} \int_{\Omega}\left(1-|u|^{2}\right)^{2}
$$

in the class

$$
\mathscr{E}_{\mathbf{d}}=\left\{u \in H^{1}(\Omega ; \mathbb{C}) ;|\operatorname{tr} u|=1 \text { on } \partial \Omega, \operatorname{deg}\left(u, \Gamma_{j}\right)=d_{j}, \forall j \in \llbracket 0, k \rrbracket\right\} .
$$

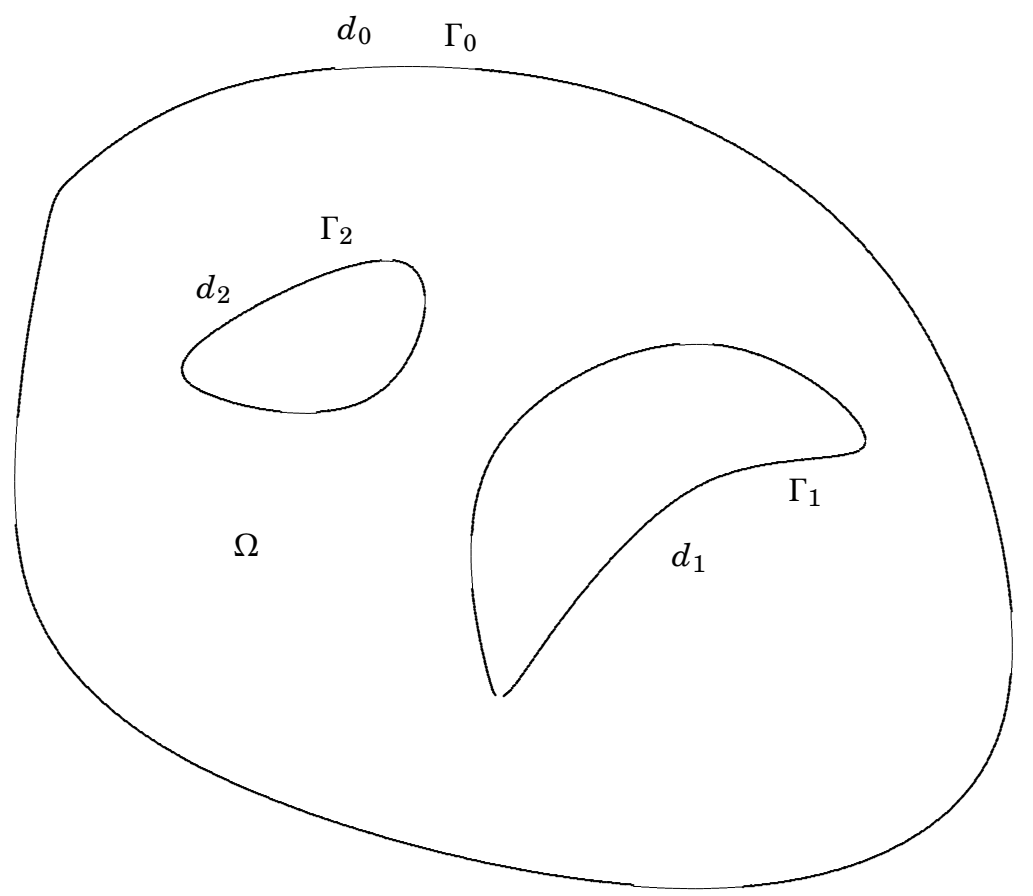

FIGURE 1 . An example with $k=2$ : $\Omega$ is a triply connected domain. The balancing condition (2.6) reads $d_{0}=d_{1}+d_{2}$. 
As in [14], the degrees on the boundary may force vortex creation. ${ }^{2}$ In contrast with the models considered in [14] or [32], the boundary condition is sufficiently flexible to let vortices approach the boundary, and we will see that, indeed, vortices sometimes do approach the boundary.

In what follows, we discuss the analysis of critical points of $E_{\varepsilon}$ in $\mathscr{E}_{\mathbf{d}}$. To start with, we will see, in Section 2, that the class $\mathscr{E}_{\mathbf{d}}$ is meaningful; this requires some explanation, since tru need not be a continuous map, and existence of the boundary degree is not obvious. We next explain, in Section 3, the non compact character of our problem; this is a major difference with [14] and [32]. ${ }^{3}$ Non compactness has consequences on existence of minimizers: in simply connected domains $\Omega$, there is no minimizer of $E_{\varepsilon}$ when $\mathbf{d} \neq 0$ (Proposition 3.3). It turns out that minimizers may exist in multiply connected domains. The first result on the existence of minimizers is due to Golovaty and Berlyand [24] and concerns thin circular annuli. In Section 4, we discuss and generalize this example, and propose a classification of domains in thin, critical and thick domains. This classification is not intrinsic (it depends on the collection d) and very likely it governs existence of minimizers; a special case was introduced in [7]. We prove asymptotic existence of minimizers in thin domains (Theorem 4.12) and discuss existence in critical domains. We conjecture that, in thick domains, minimizers of $E_{\varepsilon}$ do not exist for small $\varepsilon$. This has been established in a special case by Berlyand, Golovaty and Rybalko [5]. We discuss in Section 4 a generalization of this result. In Section 5, we account existence of critical points of $E_{\varepsilon}$ in multiply connected domains: the main result in this direction is due to Berlyand and Rybalko [11]; Dos Santos generalized their result in [22]. The case of a simply connected domain is qualitatively different. In Section 6, we present a very recent result of Berlyand, Rybalko, Sandier and the author concerning existence of critical points for large $\varepsilon$ [10], while in Section 8 we briefly describe a work in progress with Lamy on existence of critical points for small $\varepsilon$ [28]. In Section 7, we discuss two types of uniqueness results. The first one is asymptotic (Theorem 7.1), and establishes uniqueness of vortexless solutions; this generalizes previous results in [8]. The second one is non asymptotic: uniqueness is proved for each $\varepsilon$ (Theorem 7.6). This deep generalization of a previous result of Golovaty and Berlyand [24] is a very recent result of Farina and the author [23]. A final section discusses perspectives and open problems.

\section{WINDING NUMBER OF CIRCLE-VALUED MAPS}

To start with, we consider the case of a simply connected domain, say $\Omega$ is the unit disc $\mathbb{D}$. If $u \in H^{1}(\mathbb{D} ; \mathbb{C})$ is such that $|\operatorname{tr} u|=1$, then the trace $g$ of $u$ is in the class $H^{1 / 2}\left(\mathbb{S}^{1} ; \mathbb{S}^{1}\right)$. Such maps need not be continuous and existence of the degree (winding number) of $g$ has to be justified. Existence of the degree was proved by Boutet de Monvel and Gabber [16, Appendix]. This degree is defined as follows. On the one hand, $C^{\infty}\left(\mathbb{S}^{1} ; \mathbb{S}^{1}\right)$ is dense in $H^{1 / 2}\left(\mathbb{S}^{1} ; \mathbb{S}^{1}\right)$ [16, Appendix]. ${ }^{4}$ On the other hand, if we write in Fourier series $g=\sum a_{n} e^{\ln \theta}$ a smooth circle-valued map $g$, then

$$
\operatorname{deg} g=\operatorname{deg}\left(g, \mathbb{S}^{1}\right)=\sum n\left|a_{n}\right|^{2} ;
$$

this beautiful formula was discovered by Brezis and Nirenberg [20]. Since the $H^{1 / 2}$ semi-norm

$$
H^{1 / 2} \ni g \mapsto|g|_{H^{1 / 2}}^{2}:=\frac{1}{2 \pi} \int_{\mathbb{D}}|\nabla u|^{2}, \quad \text { where } u \text { is the harmonic extension of } g,
$$

is given by

$$
|g|_{H^{1 / 2}}^{2}=\sum|n|\left|a_{n}\right|^{2}
$$

we find, by combining (2.1) and (2.2), that the degree of smooth maps is continuous with respect to the $H^{1 / 2}$ convergence. This implies that the right-hand side of (2.1) is an integer for each map $g \in H^{1 / 2}\left(\mathbb{S}^{1} ; \mathbb{S}^{1}\right)$, and this integer is naturally called the degree of $g$.

\footnotetext{
${ }^{2}$ This will be indeed the case when $d_{0} \neq \sum_{j \geq 1} d_{j}$.

${ }^{3}$ Minimization of the full GL energy is also non compact, but this is only due to the gauge invariance of the GL energy, and non compactness can be removed by fixing an appropriate gauge, e.g. the Coulomb one [35]; see also [26, Chapter V].

${ }^{4}$ The point here is not density of smooth maps, but density of smooth circle-valued maps.
} 
The above implies at once existence of the degree for maps in $H^{1 / 2}\left(\Gamma ; \mathbb{S}^{1}\right)$, where $\Gamma$ is a simple closed rectifiable curve. Indeed, consider a fixed bi-Lipschitz orientation preserving homeomorphism $\Psi$ between $\Gamma$ and $\mathbb{S}^{1}$. Then we set

$$
\operatorname{deg}(g, \Gamma)=\operatorname{deg}\left(g \circ \Psi^{-1}, \mathbb{S}^{1}\right) .
$$

It is easy to see that this degree coincides with the usual one in the case of continuous maps and does not depend on the choice of $\Psi$.

This degree is a special case of the degree of VMO (vanishing mean oscillation) maps from $\mathbb{S}^{n}$ into $\mathbb{S}^{n}$, thoroughly studied by Brezis and Nirenberg [20]. It preserves most of the properties of the degree of continuous maps; see [18] or [10, Section 2] for a detailed discussion. We quote here few properties which are relevant for us.

\subsection{Proposition. Let $\Gamma$ be a smooth simple closed rectifiable curve. Then}

1. The degree of $H^{1 / 2}\left(\Gamma ; \mathbb{S}^{1}\right)$ maps is continuous with respect to strong $H^{1 / 2}$-convergence.

2. The degree of $H^{1 / 2}\left(\Gamma ; \mathbb{S}^{1}\right)$ maps is not continuous with respect to weak $H^{1 / 2}$-convergence.

3. $\operatorname{deg}(g h)=\operatorname{deg} g+\operatorname{deg} h, \forall g, h \in H^{1 / 2}\left(\Gamma ; \mathbb{S}^{1}\right)$.

4. A map $g \in H^{1 / 2}\left(\Gamma ; \mathbb{S}^{1}\right)$ can be written as $g=e^{\imath \psi}$ with $\psi \in H^{1 / 2}(\Gamma ; \mathbb{R})$ if and only if $\operatorname{deg} g=0$.

From the above, the class $\mathscr{E}_{\mathbf{d}}$ defined in the introduction is meaningful, provided we precise the orientation on each component $\Gamma_{j}$ of $\partial \Omega$. The convention we use here is that each $\Gamma_{j}$ is endowed with the natural (counterclockwise) orientation. Thus, if $\Omega=\mathbb{D} \backslash \mathbb{D}_{1 / 2},{ }^{5}$ then $z \mapsto\left(\frac{z}{|z|}\right)^{d}$ belongs to the class $\mathscr{E}(d, d)$.

Once critical points of $E_{\varepsilon}$ in $\mathscr{E}_{\mathbf{d}}$ are obtained, we may forget the generalized degree. Indeed, we have the following result [8, Lemma 4.4].

2.2. Proposition. Assume that $\Omega$ is smooth. Let $u$ be a critical point of $E_{\varepsilon}$ in $\mathscr{E}_{\mathbf{d}}$. Then $u \in C^{\infty}(\bar{\Omega})$, and criticality is equivalent to either the strong form

$$
\left\{\begin{aligned}
-\Delta u & =\frac{1}{\varepsilon^{2}} u\left(1-|u|^{2}\right) & & \text { in } \Omega \\
|\operatorname{tr} u| & =1 & & \text { on } \partial \Omega \\
u \wedge \frac{\partial u}{\partial v} & =0 & & \text { on } \partial \Omega \\
\operatorname{deg}\left(u, \Gamma_{j}\right) & =d_{j} & & \forall j \in \llbracket 0, k \rrbracket
\end{aligned}\right.
$$

or the weak form

$$
\left\{\begin{array}{rlrl}
-\Delta u & =\frac{1}{\varepsilon^{2}} u\left(1-|u|^{2}\right) & & \text { in } \Omega \\
|\operatorname{tr} u| & =1 & & \text { on } \partial \Omega \\
\int_{\Omega}(u \wedge \nabla u) \cdot \nabla \zeta & =0 & & \forall \zeta \in H^{1}(\Omega) \\
\operatorname{deg}\left(u, \Gamma_{j}\right) & =d & & \forall j \in \llbracket 0, k \rrbracket
\end{array} .\right.
$$

Here, $\wedge$ stands for the vector product of complex numbers: $\left(a_{1}+\imath a_{2}\right) \wedge\left(b_{1}+\imath b_{2}\right)=a_{1} b_{2}-a_{2} b_{1}$. Similarly, the notation $u \wedge \nabla v$, with $u$ and $v$ complex-valued functions, denotes the vector-field $u_{1} \nabla v_{2}-u_{2} \nabla v_{1}$.

We end this section by discussing a first vortex creation mechanism. If $g \in C\left(\mathbb{D} ; \mathbb{S}^{1}\right)$, then $g$ has a non vanishing (or, equivalently, circle-valued) continuous extension $u$ on $\overline{\mathbb{D}}$ if and only if deg $g=0$. In a multiply connected domain $\Omega$, the necessary and sufficient condition for the existence of a non vanishing continuous extension is the balancing condition

$$
\operatorname{deg}\left(g, \Gamma_{0}\right)=d_{0}=\sum_{j \geq 1} d_{j}=\sum_{j \geq 1} \operatorname{deg}\left(g, \Gamma_{j}\right) .
$$

This extends to $H^{1}$ maps [17].

\footnotetext{
${ }^{5}$ We denote by $\mathbb{D}_{r}$ the disc of radius $r$ centered at the origin, and by $C_{r}$ the circle of radius $r$ then the map centered at the origin.
} 
2.3. Proposition. Let $\Omega \subset \mathbb{R}^{2}$ be a smooth bounded domain. For $g \in H^{1 / 2}\left(\partial \Omega ; \mathbb{S}^{1}\right)$, the following are equivalent.

1. g has an extension $u \in H^{1}\left(\Omega ; \mathbb{S}^{1}\right)$.

2. $g$ has an extension $u \in H^{1}(\Omega ; \mathbb{C})$ such that $|u| \geq C>0$.

3. The balancing condition (2.6) holds.

2.4. Definition. $\mathbf{d} \in \mathbb{Z}^{k+1}$ is balanced if $d_{0}=\sum_{j \geq 1} d_{j}$, and unbalanced otherwise. By extension, a domain $\Omega$ (with prescribed degrees $\mathbf{d}=\left(d_{0}, \ldots, d_{k}\right)$ ) is balanced if $\mathbf{d}$ is balanced, unbalanced otherwise.

\section{NON COMPACTNESS}

The fact that the class $\mathscr{E}_{\mathbf{d}}$ is not weakly closed is essentially equivalent to Proposition 2.12 , and relies on the following fundamental example.

3.1. Lemma. Let $\mathbf{d} \in \mathbb{Z}^{k+1}$. Then there exists a sequence $\left(u_{n}\right) \subset \mathscr{E}_{\mathbf{d}}$ such that

$$
\left|u_{n}\right| \leq 1, u_{n} \rightarrow 1 \text { and } \int_{\Omega}\left|\nabla u_{n}\right|^{2} \rightarrow 2 \pi|\mathbf{d}| \text {. }
$$

In particular, we have

$$
\mathbf{m}_{\mathbf{d}, \varepsilon}:=\inf _{\mathscr{E}_{\mathbf{d}}} E_{\varepsilon}(u) \leq \pi|\mathbf{d}| .
$$

Here and in what follows, the length $|\mathbf{d}|$ is defined by

$$
|\mathbf{d}|=\sum_{j=0}^{k}\left|d_{j}\right| .
$$

Proof. For $j \in \llbracket 1, k \rrbracket$, let $\omega_{j}$ denote the exterior of $\Gamma_{j}$, and let $\omega_{0}$ denote the interior of $\Gamma_{0}$, so that $\Omega=\bigcap_{j \geq 0} \omega_{j}$. Fix a conformal representation $\Phi_{j}: \omega_{j} \rightarrow \mathbb{D}$ of $\omega_{j}$ into the unit disc $\mathbb{D}$. Let

$$
M_{\alpha, a}(z):=\alpha \frac{z-a}{1-\bar{a} z}, \forall \alpha \in \mathbb{S}^{1}, a, z \in \mathbb{D}, \quad M_{a}:=M_{1, a}
$$

be the Moebius transforms. Let $v_{j, a}=M_{a} \circ \Phi_{j}, a \in \mathbb{D}, j \in \llbracket 0, k \rrbracket$. Then $\left|v_{j, a}\right|=1$ on $\Gamma_{j}$ and $\operatorname{deg}\left(v_{j, a}, \Gamma_{j}\right)=1$. In addition, we have

$$
\int_{\Omega}\left|\nabla v_{j, a}\right|^{2} \rightarrow 2 \pi \text { and } v_{j, a} \rightarrow 1 \text { in } C_{l o c}^{\infty}\left(\bar{\Omega} \backslash\left\{\Phi^{-1}(-1)\right\}\right) \text { as } a \rightarrow-1 \text {. }
$$

Using (3.4), it is easy to modify $v_{j, a}$ near $\partial \Omega \backslash \Gamma_{j}$ and construct a map $u_{j, a}$ with the following properties:

$$
\begin{aligned}
& u_{j, a} \in H^{1}(\Omega ; \mathbb{C}),\left|u_{j, a}\right| \leq 1,\left|\operatorname{tr} u_{j, a}\right|=1, \operatorname{deg}\left(u_{j, a}, \Gamma_{l}\right)= \begin{cases}1, & \text { if } l=j \\
0, & \text { if } l \neq j\end{cases} \\
& \int_{\Omega}\left|\nabla u_{j, a}\right|^{2} \rightarrow 2 \pi \text { and } u_{j, a} \rightarrow 1 \text { in } C_{l o c}^{k}\left(\bar{\Omega} \backslash\left\{\Phi^{-1}(-1)\right\}\right) \text { as } a \rightarrow-1 .
\end{aligned}
$$

If we now let

$$
u_{n}=\prod_{j=0}^{k} u_{a, j}^{d_{j}}, \quad \text { where } a=a(n) \rightarrow-1 \text { as } n \rightarrow \infty,
$$

it is easy to see that $u_{n}$ has all the required properties. ${ }^{6}$

3.2. Corollary. The class $\mathscr{E}_{\mathbf{d}}$ is not weakly closed. More specifically, the weak sequential closure of $\mathscr{E}_{\mathbf{d}}$ is $\bigcup_{\mathbf{e} \in \mathbb{Z}^{k+1}} \mathscr{E}_{\mathbf{e}}=\left\{u \in H^{1}(\Omega ; \mathbb{C}) ;|\operatorname{tr} u|=1\right\}$.

\footnotetext{
${ }^{6}$ Here, we use the convention $u^{-l}=(\bar{u})^{l}$ if $l \in \mathbb{N}$.
} 
Proof. Clearly, the weak closure is contained in $\bigcup_{\mathbf{e} \in \mathbb{Z}^{k+1}} \mathscr{E}_{\mathbf{e}}$. Conversely, let $u \in \mathscr{E}_{\mathbf{e}}$. Let $\left(u_{n}\right) \subset \mathscr{E}_{\mathbf{d}-\mathbf{e}}$ be as in Lemma 3.1. Then $\left(u u_{n}\right) \subset \mathscr{E}_{\mathbf{d}}$ and $u u_{n} \rightarrow u$.

Corollary 3.2 implies that existence of a minimizer of $E_{\varepsilon}$ in $\mathscr{E}_{\mathbf{d}}$ cannot be obtained by a straightforward application of the direct method in the calculus of variations, since a bounded sequence in $\mathscr{E}_{\mathbf{d}}$ need not converge to a a map in $\mathscr{E}_{\mathbf{d}}$. However, by itself, Corollary 3.2 does not imply that the minimum of $E_{\varepsilon}$ is not attained in $\mathscr{E}_{\mathbf{d}}{ }^{7}$ Here is an example of non existence of minimizer [8].

3.3. Proposition. Assume that all the $d_{j}$ 's but one are zero. Then

(3.7) $\inf _{u \in \mathscr{E}_{\mathbf{d}}} E_{\varepsilon}(u)=\pi|\mathbf{d}|$,

and the infimum is not attained in (3.7).

Proof. We consider, e.g., the case where $\mathbf{d}=(d, 0, \ldots, 0)$, with $d>0$; the other cases are similar. By Lemma 3.1, we have $\leq$ in (3.7). On the other hand, we have the pointwise inequality

(3.8) $|\nabla u|^{2} \geq 2$ Jac $u$.

This implies that

$$
E_{\varepsilon}(u) \geq \int_{\Omega} \operatorname{Jac} u+\frac{1}{4 \varepsilon^{2}} \int_{\Omega}\left(1-|u|^{2}\right)^{2}=\pi d+\frac{1}{4 \varepsilon^{2}} \int_{\Omega}\left(1-|u|^{2}\right)^{2},
$$

the last equality following from the identity

$$
\int_{\Omega} \operatorname{Jac} u=\pi\left(d_{0}-\sum_{j \geq 1} d_{j}\right),
$$

classically valid for smooth maps and still valid when $u \in \mathscr{E}_{\mathbf{d}}$ [18].

In particular, (3.9) implies $\geq$ (and thus $=$ ) in (3.7).

Now argue by contradiction and assume that the infimum is attained in (3.7). If $u$ is a minimizer, then, by (3.9), $|u|=1$ a.e. By Proposition Proposition 2.3, this implies $d=0$, a contradiction.

It turns out that the above example is the only known one where the problem of existence of a minimizer can be settled via a simple argument.

\section{SizE OF DOMAINS}

In this section, we introduce appropriate definitions of thin/thick domains, relevant for existence of minimizers of $E_{\varepsilon}$ in $\mathscr{E}_{\mathbf{d}}$; special cases of this classification were considered in the work [7] devoted to doubly connected domains. These definitions involve the value of the infimum of $E_{\varepsilon}$ in some classes of circle-valued maps.

To start with, let us denote by $\mathscr{B}$ the collection of balanced degrees:

$$
\mathscr{B}=\left\{\mathbf{d} \in \mathbb{Z}^{k+1} ; d_{0}=\sum_{j \geq 1} d_{j}\right\} .
$$

When $\mathbf{d} \in \mathscr{B}$, there are circle-valued competitors in the class $\mathscr{E}_{\mathbf{d}}$ (Proposition 2.3), and thus we may consider the nonempty subclass

$$
\mathscr{F}_{\mathbf{d}}=\left\{u \in \mathscr{E}_{\mathbf{d}} ;|u|=1 \text { in } \Omega\right\} .
$$

We clearly have the $a$ priori bound

$$
\mathbf{m}_{\mathbf{d}, \varepsilon}:=\inf _{u \in \mathscr{E}_{\mathbf{d}}} E_{\varepsilon}(u) \leq \inf _{u \in \mathscr{F} \mathbf{d}} E_{\varepsilon}(u)=\inf _{u \in \mathscr{F} \mathbf{d}} \frac{1}{2} \int_{\Omega}|\nabla u|^{2}:=\mathbf{m}_{\mathbf{d}}
$$

It turns out that, in the definition of $\mathbf{m}_{\mathbf{d}}$, the infimum is actually a minimum. The following is a rephrasing of [14, Chapter 1$]$.

\footnotetext{
${ }^{7}$ Actually, the minimum is attained in some cases, as we will see later.
} 
4.1. Proposition. Fix $k$ points $a_{j} \in \omega_{j}, j \in \llbracket 1, k \rrbracket$ and consider the reference map $u_{\mathbf{d}}(z)=\prod_{j=1}^{k}\left(\frac{z-a_{j}}{\left|z-a_{j}\right|}\right)^{d_{j}}$. Then

1. $\mathscr{F}_{\mathbf{d}}=\left\{u_{\mathbf{d}} e^{\imath \psi} ; \psi \in H^{1}(\Omega ; \mathbb{R})\right\}$.

2. The minimum of the Dirichlet integral in $\mathscr{F}_{\mathbf{d}}$ is attained by $u_{\mathbf{d}} e^{\imath \psi}$, where $\psi$ is the (unique modulo constants) solution of

$$
\left\{\begin{array}{ll}
\Delta \psi=0 & \text { in } \Omega \\
\frac{\partial \psi}{\partial v}=-u_{\mathbf{d}} \wedge \frac{\partial u_{\mathbf{d}}}{\partial v} & \text { on } \partial \Omega
\end{array} .\right.
$$

Using (4.2), it is possible to compute numerically the value $\mathbf{m}_{\mathbf{d}}$. In a very special case, it is possible to compute explicitly $\mathbf{m}_{\mathbf{d}}$ [8]. This classification involves only the conformal ratio of $\Omega$, that is the unique $R>1$ such that $\Omega$ can be represented conformally onto $\mathbb{D}_{R} \backslash \overline{\mathbb{D}}$.

4.2. Proposition. Let $\Omega$ be doubly connected. Let $R$ be the conformal ratio of $\Omega$. Then $\mathbf{m}_{\mathbf{d}}=$ $\pi d_{0}^{2} \ln R$.

Proof. In this case, we have $\mathbf{d}=\left(d_{0},-d_{0}\right)$. Since the value of $\mathbf{m}_{\mathbf{d}}$ is clearly a conformal invariant, we may assume that $\Omega=\mathbb{D}_{R} \backslash \overline{\mathbb{D}}$. In this case, we pick $a_{1}=0$ and find that $\psi=0$ is solution of (4.2). Thus $u_{\mathbf{d}}$ attains the value $\mathbf{m}_{\mathbf{d}}$, and clearly $\int_{\Omega}\left|\nabla u_{\mathbf{d}}\right|^{2}=2 \pi d_{0}^{2} \ln R$.

We next give some simple but fundamental upper bounds for $\mathbf{m}_{\mathbf{d}, \varepsilon}$.

4.3. Proposition. We have

$$
\mathbf{m}_{\mathbf{d}, \varepsilon} \leq \mathbf{m}_{\mathbf{e}, \varepsilon}+\pi|\mathbf{d}-\mathbf{e}|, \quad \forall \mathbf{e} \in \mathbb{Z}^{k+1}
$$

and

(4.4) $\quad \mathbf{m}_{\mathbf{d}, \varepsilon} \leq \mathbf{m}_{\mathbf{e}}+\pi|\mathbf{d}-\mathbf{e}|, \quad \forall \mathbf{e} \in \mathscr{B}$.

Proof. Let $v \in \mathscr{E}_{\mathbf{d}}$. Assume that the following holds:

$$
|v| \leq 1 \text { and } \lim _{z \rightarrow \partial \Omega}|v(z)|=1 \text { uniformly. }
$$

Consider a sequence $u_{n} \in \mathscr{E}_{\mathbf{e}-\mathbf{d}}$ as in Lemma 3.1. Then $\left(v u_{n}\right) \subset \mathscr{E}_{\mathbf{e}}$ and (by (3.5), (3.6) and (4.5)) $E_{\varepsilon}\left(v u_{n}\right)=E_{\varepsilon}(v)+\pi|\mathbf{d}-\mathbf{e}|+o(1)$. We find that

(4.6) $\inf \left\{E_{\varepsilon}(v) ; v \in \mathscr{E}_{\mathbf{d}}, v\right.$ satisfies $\left.(4.5)\right\} \leq \mathbf{m}_{\mathbf{e}, \varepsilon}+\pi|\mathbf{d}-\mathbf{e}|$.

We next argue as follows: given $u \in \mathscr{E}_{\mathbf{d}}$, let $v$ minimize $E_{\varepsilon}$ in the class $\left\{w \in H^{1}(\Omega)\right.$; $\left.\operatorname{tr} w=\operatorname{tr} u\right\}$. Then we have $v \in \mathscr{E}_{\mathbf{d}}$ and $E_{\varepsilon}(u) \leq E_{\varepsilon}(v)$. On the other hand, this $v$ satisfies (4.5). Indeed, the first property in (4.5) follows from the maximum principle, while the second one is essentially due to Boutet de Monvel and Gabber [16, Appendix]; see also [21, Theorem A.3.2] and [10, Section 2].

Therefore, the left-hand side of (4.6) equals $\mathbf{m}_{\mathbf{d}, \varepsilon}$, and this implies (4.3).

Estimate (4.4) follows by combining (4.3) with (4.1) (applied with $\mathbf{e} \in \mathscr{B}$ ).

Bound (4.3) suggests the following result, very much in the spirit of the famous conditions of Trudinger [34] or Aubin [3] for the existence of solutions of the Yamabe problem .

\subsection{Proposition. Assume that}

$$
\mathbf{m}_{\mathbf{d}, \varepsilon}<\mathbf{m}_{\mathbf{e}, \varepsilon}+\pi|\mathbf{d}-\mathbf{e}|, \quad \forall \mathbf{e} \in \mathbb{Z}^{k+1} \backslash\{\mathbf{d}\} .
$$

Then minimizing sequences for $E_{\varepsilon}$ in $\mathscr{E}_{\mathbf{d}}$ are compact in $H^{1}$.

Proof. We rely on the following "converse" to Lemma 3.1 [7].

4.5. Lemma (Price lemma). Let $\left(u_{n}\right) \subset \mathscr{E}_{\mathbf{d}}$ be such that $u_{n} \rightarrow u \in \mathscr{E} \mathbf{e}$. Then

$$
\liminf \frac{1}{2} \int_{\Omega}\left|\nabla u_{n}\right|^{2} \geq \frac{1}{2} \int_{\Omega}|\nabla u|^{2}+\pi|\mathbf{d}-\mathbf{e}|
$$


Proof of the Price lemma. We present the proof under the additional assumption that $u_{n}$ and $u$ are smooth. This is not crucial for the analysis, but simplifies the proof. ${ }^{8}$ The starting point is provided by the identities ${ }^{9}$

$$
\int_{\Omega} f \operatorname{Jac} v=\int_{\Omega}\left(\partial_{x} f \partial_{y} v \wedge v-\partial_{y} f \partial_{x} v \wedge v\right)+\int_{\partial \Omega} f v \wedge \frac{\partial v}{\partial \tau}, \quad \forall v \in C^{1}(\bar{\Omega} ; \mathbb{C}), \forall f \in C^{1}(\bar{\Omega} ; \mathbb{R})
$$

and

$$
\operatorname{deg}\left(g, \Gamma_{j}\right)=\frac{1}{2 \pi} \int_{\Gamma_{j}} g \wedge \frac{\partial g}{\partial \tau}, \quad \forall g \in C^{1}\left(\Gamma_{j} ; \mathbb{S}^{1}\right),
$$

and by the fact that, by standard properties of weakly convergent sequences and by (3.8), we have

$$
\begin{aligned}
\int_{\Omega}\left|\nabla u_{n}\right|^{2} & =\int_{\Omega}|\nabla u|^{2}+\int_{\Omega} \mid \nabla(\left.\underbrace{u_{n}-u}_{v_{n}}\right|^{2}+o(1) \geq \int_{\Omega}|\nabla u|^{2}+\int_{\Omega}|f|\left|\nabla v_{n}\right|^{2}+o(1) \\
& \geq \int_{\Omega}|\nabla u|^{2}+2 \int_{\Omega} f \operatorname{Jac} v_{n}+o(1), \quad \forall f \in C^{1}(\bar{\Omega} ;[-1,1]) .
\end{aligned}
$$

By combining (4.11) with (4.9) (applied with $v=v_{n}$ ) we find that

$$
\liminf \int_{\Omega}\left|\nabla u_{n}\right|^{2} \geq \int_{\Omega}|\nabla u|^{2}+2 \liminf \int_{\partial \Omega} f v_{n} \wedge \frac{\partial v_{n}}{\partial \tau}, \quad \forall f \in C^{1}(\bar{\Omega} ;[-1,1]) .
$$

We now take $f \in C^{1}(\bar{\Omega} ;[-1,1])$ such that $f=\operatorname{sgn}\left(d_{j}-e_{j}\right)$ on $\Gamma_{j}$. For such $f$, trace theory for $H^{1}$ maps combined with weak convergence of $\left(u_{n}\right)$ to $u$ and with formula (4.10) yields

$$
\lim \int_{\partial \Omega} f v_{n} \wedge \frac{\partial v_{n}}{\partial \tau}=\lim \int_{\partial \Omega} f\left(u_{n} \wedge \frac{\partial u_{n}}{\partial \tau}-u \wedge \frac{\partial u}{\partial \tau}\right)=2 \pi \sum_{j}\left|d_{j}-e_{j}\right|=2 \pi|\mathbf{d}-\mathbf{e}| \text {. }
$$

Proof of Proposition 4.4 completed. Let $\left(u_{n}\right)$ be a minimizing sequence for $E_{\varepsilon}$ in $\mathscr{E}_{\mathbf{d}}$. Let $u$ be such, possibly up to a subsequence, $u_{n} \rightarrow u \in \mathscr{E}_{\mathbf{e}}$. If we prove that $\mathbf{e}=\mathbf{d}$, then $u_{n} \rightarrow u$, since the sequence $\left(u_{n}\right)$ is minimizing. The conclusion $\mathbf{e}=\mathbf{d}$ is obtained by contradiction: otherwise, we have, by the Price lemma,

$$
\mathbf{m}_{\mathbf{d}, \varepsilon}=\lim E_{\varepsilon}\left(u_{n}\right) \geq E_{\varepsilon}(u)+\pi|\mathbf{d}-\mathbf{e}| \geq \mathbf{m}_{\mathbf{e}, \varepsilon}+\pi|\mathbf{d}-\mathbf{e}|,
$$

and this contradicts (4.7).

In order to be able to apply Proposition 4.4, we need tractable conditions implying (4.7). This is where our classification of domaines comes into the picture.

4.6. Definition. Let $\Omega$ be a domain with associated degrees $\mathbf{d}$. If $\mathbf{d}$ is unbalanced, then $\Omega$ is thick. If $\mathbf{d}$ is balanced, then:

1. $\Omega$ is thin provided

$$
\mathbf{m}_{\mathbf{d}}<\mathbf{m}_{\mathbf{e}}+\pi|\mathbf{d}-\mathbf{e}|, \quad \forall \mathbf{e} \in \mathscr{B} \backslash\{\mathbf{d}\} .
$$

2. $\Omega$ is critical when

$$
\mathbf{m}_{\mathbf{d}}=\min \left\{\mathbf{m}_{\mathbf{e}}+\pi|\mathbf{d}-\mathbf{e}|, \mathbf{e} \in \mathscr{B} \backslash\{\mathbf{d}\}\right\} .
$$

3. $\Omega$ is thick in the remaining cases, i.e. when

$$
\mathbf{m}_{\mathbf{d}}>\mathbf{m}_{\mathbf{e}}+\pi|\mathbf{d}-\mathbf{e}| \quad \text { for some } \mathbf{e} \in \mathscr{B} \backslash\{\mathbf{d}\} .
$$

\footnotetext{
${ }^{8}$ The identities we derive in the proof of the Price lemma can be extended to arbitrary $u_{n}$ and $u$; see [7].

${ }^{9}$ Here, $\partial \tau$ stands for the tangential derivative.
} 
At first look, the classification involves an infinite numbers of inequalities. However, it is easy to see that, for a fixed $\Omega$, we have

(4.17) $\lim _{|\mathbf{e}| \rightarrow \infty} \mathbf{m}_{\mathbf{e}}=\infty$,

and thus, when $\mathbf{d}$ is balanced, $\Omega$ is thin/critical/thick provided

(4.18) $\mathbf{m}_{\mathbf{d}}<$ or $=$ or $>\min \left\{\mathbf{m}_{\mathbf{e}}+\pi|\mathbf{d}-\mathbf{e}|, \mathbf{e} \in \mathscr{B} \backslash\{\mathbf{d}\}\right\}$.

First proof of (4.17). Argue by contradiction and assume that, along a sequence $\left(\mathbf{e}^{l}\right)$ such that $\left|\mathbf{e}^{l}\right| \rightarrow \infty$, we have $\mathbf{m}_{\mathbf{e}^{l}} \leq C$. Assume, e.g., that (again possibly up to a subsequence), $\left|\mathbf{e}_{0}^{l}\right| \rightarrow \infty .{ }^{10}$ Let $u^{l} \in \mathscr{E}_{\mathbf{e}^{l}}$ be such that $\int_{\Omega}\left|\nabla u^{l}\right|^{2} \leq C$. By a standard procedure, we may extend $u^{l}$ to a map $v^{l} \in H^{1}\left(\omega_{0} ; \mathbb{C}\right)$ such that $\int_{\Omega}\left|\nabla v^{l}\right|^{2} \leq C^{\prime}$ and $\left|v^{l}\right| \leq 1$. Thus $v^{l} \in \mathscr{E}_{\mathbf{e}_{0}^{l}}$ and $E_{1}\left(v^{l}\right) \leq C^{\prime \prime}$. This contradicts, for large $l$, (3.7).

4.7. Remark. The same analysis implies the following: if $u^{l} \in \mathscr{E}_{\mathbf{e}^{l}}$ and $\varepsilon^{l}>0$ are such that $E_{\varepsilon^{l}}\left(u^{l}\right) \leq$ $C$, then $\left(\mathbf{e}^{l}\right)$ is bounded.

Second proof of (4.17). Let, for $j \in \llbracket 1, k \rrbracket$, the collection $\mathbf{d}^{j}$ given by $\mathbf{d}_{k}^{j}=\left\{\begin{array}{ll}1, & \text { if } k=0 \text { or } j \\ 0, & \text { otherwise }\end{array}\right.$. Let $u^{j}$ minimize $\mathbf{m}_{\mathbf{d}^{j}}$. Using Proposition 4.1 , it is easy yo see that $u=\prod_{j=1}^{k}\left(u^{j}\right)^{e_{j}} \operatorname{minimizes} \mathbf{m}_{\mathbf{e}}$. Thus $\mathbf{m}_{\mathbf{e}}=\frac{1}{2}(A \mathbf{e}) \cdot \mathbf{e}$, where $A$ is the definite positive Gramm matrix $A=\left(\int_{\Omega} \nabla u^{j} \cdot \nabla u^{l}\right)_{j, l \in \llbracket 1, k \rrbracket}$. We find that $\mathbf{m}_{\mathbf{e}} \rightarrow \infty$ as $|\mathbf{e}| \rightarrow \infty$.

Before going further, let us discuss some examples.

The first two ones are trivial and do not lead to any interesting consequence. First, every domain is thin with respect to the trivial collection $\mathbf{d}=0$. Next, a simply connected domain is thick with respect to any non trivial collection (since it is unbalanced).

We next classify doubly connected domains. Let $\mathbf{d}=(d,-d)$ be a non trivial balanced collection. With no loss of generality, we may assume that $d>0$. The next result is a straightforward consequence of Proposition 4.2.

4.8. Proposition. Let $\Omega$ be doubly connected of conformal ratio $R$. Let $\mathbf{d}=(d,-d)$, with $d>0$. Then:

1. $\Omega$ is thin when $R<e^{2 /(2 d-1)}$.

2. $\Omega$ is critical when $R=e^{2 /(2 d-1)}$.

3. $\Omega$ is thick when $R>e^{2 /(2 d-1)}$.

4.9. Remark. This shows clearly that the size of a domain is not intrinsic: it depends both on the domain and on the collection $\mathbf{d}$.

Proposition 4.8 implies that our classification of domains coincides, in the special case where $\Omega$ is doubly connected and $\mathbf{d}=(1,-1)$, with the one considered in [7].

We next give an example involving triply connected domains. Consider a domain $\Omega_{t}$ as in Fig. 2 and let $\mathbf{d} \in \mathscr{B} \backslash\{0\}$ be any non trivial balanced collection.

Then we have the following

4.10. Proposition. There exist two values $0<t_{0}=t_{0}(\mathbf{d}) \leq t_{1}=t_{1}(\mathbf{d})<1$ such that:

1. For $t<t_{0}, \Omega_{t}$ is thick.

2. For some $t \in\left[t_{0}, t_{1}\right], \Omega_{t}$ is critical.

3. For $t>t_{1}, \Omega_{t}$ is thin.

\footnotetext{
${ }^{10}$ The case where $\left|\mathbf{e}_{j}^{l}\right| \rightarrow \infty$ for some $j \geq 1$ is reduced to the case $j=0$ via the an inversion, using the invariance of the Dirichlet integral with respect to conformal changes of variables.
} 
Proof. Let $z^{1}=(-1 / 2,0)$ and $z^{2}=(1 / 2,0)$ be the centers of the two squares. By considering the test function

$$
u(z)=\left(\frac{z-z^{1}}{\left|z-z^{1}\right|}\right)^{d_{1}}\left(\frac{z-z^{2}}{\left|z-z^{2}\right|}\right)^{d_{2}} \in \mathscr{E}_{\mathbf{d}},
$$

it is easy to see that $\mathbf{m}_{\mathbf{d}} \rightarrow 0$ as $t \rightarrow 1$. Since $\mathbf{m}_{\mathbf{e}}+\pi|\mathbf{d}-\mathbf{e}| \geq 2 \pi$ for $\mathbf{e} \neq \mathbf{d}$, this implies 3 .

In order to obtain 1 , it suffices to prove that

(4.19) $\mathbf{m}_{\mathbf{d}} \rightarrow \infty$ as $t \rightarrow 0$,

and thus

$$
\mathbf{m}_{\mathbf{d}}<\mathbf{m}_{0}+\pi|\mathbf{d}-0|=\pi|\mathbf{d}|
$$

for small $t$.

Now (4.19) is obtained as follows: let $A_{t}=\mathbb{D} \backslash \mathbb{D}_{t}$ and $B_{t}=(-1 / 2,1 / 2)^{2} \backslash(-t / 2, t / 2)^{2}, t \in(0,1)$. Let $\Phi: \mathbb{S}^{1} \rightarrow \partial\left[(-1 / 2,1 / 2)^{2}\right]$ be a bi-Lispchitz map. By considering the bi-Lispchitz map

$$
z=\left(z_{1}, z_{2}\right) \mapsto|z| \Phi\left(\frac{z}{|z|}\right),
$$

which maps $A_{t}$ into $B_{t}$, we see that, with obvious notations, we have

(4.20) $\mathbf{m}_{\mathbf{d}}\left(A_{t}\right) \sim \mathbf{m}_{\mathbf{d}}\left(B_{t}\right)$.

We obtain (4.19) by combining (4.20) with Proposition 4.2 and with the obvious inequality

$$
\mathbf{m}_{\mathbf{d}}\left(\Omega_{t}\right) \geq \mathbf{m}_{\left(d_{1},-d_{1}\right)}\left(B_{t}\right)+\mathbf{m}_{\left(d_{2},-d_{2}\right)}\left(B_{t}\right) .
$$

Finally, let

$$
t_{2}=\sup \left\{s \in(0,1) ; \Omega_{t} \text { is thin for every } t \in(0, s)\right\} \in\left[t_{0}, t_{1}\right] .
$$

Using again a bi-Lipschitz change of variables argument, it is clear that the maps $t \mapsto \mathbf{m}_{\mathbf{e}}\left(\Omega_{t}\right)$ are continuous (and non increasing) for every $\mathbf{e} \in \mathscr{B}$. Using this fact combined with (4.17), it is easy to see that $\Omega_{t_{2}}$ is critical.

4.11. Remark. More can be said in the special case where $\Omega=\Omega_{t}$ and $\mathbf{d}=(2 d, d, d)$ with $d \neq 0$. It is easy to see that $\mathscr{E}_{(2 d, d, d)}=\left\{u^{d} ; u \in \mathscr{E}_{(2,1,1)}\right\}$ and therefore $\mathbf{m}_{(2 d, d, d)}=d^{2} \mathbf{m}_{(2,1,1)}$.

On the other hand, the map $t \mapsto \mathbf{m}_{\mathbf{d}}\left(\Omega_{t}\right)$ is better than non increasing: it is decreasing. Using the above, we easily find that there exists a unique threshold value $t_{0}=t_{1}$ which makes $\Omega_{t}$ switch from thin for $t>t_{0}$ to thick when $t<t_{0}$. More precisely, the value $t_{0}$ is the solution of $\mathbf{m}_{(2,1,1)}\left(\Omega_{t}\right)=$ $\frac{4 \pi}{2|d|-1}$.

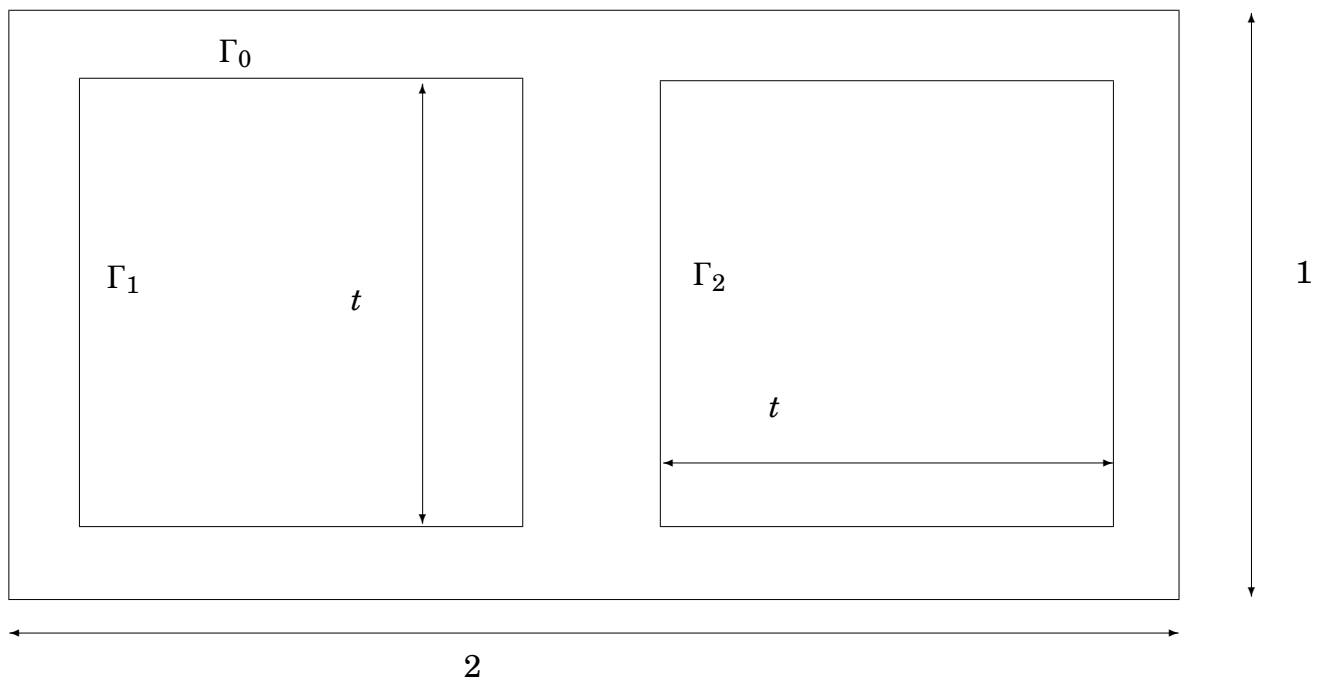

FIGURE 2. $\Omega_{t}$ is the rectangle $(-1,1) \times(-1 / 2,1 / 2)$ perforated by two squares of size $t \in(0,1)$ centered at $( \pm 1 / 2,0)$. 
We may now state a first tractable condition for the existence of minimizers of $\mathbf{m}_{\mathbf{d}, \varepsilon}$.

4.12. Theorem. Assume that $\Omega$ is thin. Then, for small $\varepsilon$, the infimum $\mathbf{m}_{\mathbf{d}, \varepsilon}$ of $E_{\varepsilon}$ in $\mathscr{E}_{\mathbf{d}}$ is attained.

Proof. Let $\left(u_{n, \varepsilon}\right)$ be a minimizing sequence for $E_{\varepsilon}$ in $\mathscr{E}_{\mathbf{d}}$ and let $u_{\varepsilon}$ be such that, possibly up to a subsequence, $u_{n, \varepsilon} \rightarrow u$. Let $\mathbf{e} \in \mathbb{Z}^{k+1}$ be such that $u_{\varepsilon} \in \mathscr{E}_{\mathbf{e}}$. Using the upper bound

(4.21) $\mathbf{m}_{\mathbf{d}, \varepsilon} \leq \pi|\mathbf{d}|$

(which follows from Lemma 3.1) and Remark 4.7, we may assume that, possibly up to a subsequence, $\mathbf{e}$ is independent of small $\varepsilon$. Using (4.21), we find that, up to a subsequence, $u_{\varepsilon} \rightarrow u$ to some $u \in H^{1}(\Omega)$. In addition, we have $\frac{1}{4 \varepsilon^{2}}\left(1-\left|u_{\varepsilon}\right|^{2}\right)^{2} \leq \pi|\mathbf{d}|$, and this implies that $u$ is circle-valued. Let $\mathbf{f} \in \mathbb{Z}^{k+1}$ be such that $u \in \mathscr{O}_{\mathbf{f}}$. Since $u$ is circle-valued, we find that $\mathbf{f}$ is balanced. We now apply twice the Price lemma. Using the a priori bound (4.1), we find that

$$
\begin{aligned}
\mathbf{m}_{\mathbf{d}} \geq \lim _{\varepsilon \rightarrow 0} \mathbf{m}_{\mathbf{d}, \varepsilon} & =\lim _{\varepsilon \rightarrow 0} \lim _{n} E_{\varepsilon}\left(u_{n, \varepsilon}\right) \geq \underset{\varepsilon \rightarrow 0}{\limsup }\left(\frac{1}{2} \int_{\Omega}\left|\nabla u_{\varepsilon}\right|^{2}+\pi|\mathbf{d}-\mathbf{e}|\right) \\
& \geq \frac{1}{2} \int_{\Omega}|\nabla u|^{2}+\pi(|\mathbf{d}-\mathbf{e}|+|\mathbf{e}-\mathbf{f}|) \geq \mathbf{m}_{\mathbf{f}}+\pi(|\mathbf{d}-\mathbf{e}|+|\mathbf{e}-\mathbf{f}|) .
\end{aligned}
$$

Using the fact that $\Omega$ is thin, we find that $\mathbf{d}=\mathbf{e}=\mathbf{f}$. In particular, we proved that, for small $\varepsilon$, minimizing sequences are compact.

In some special situations, it is possible to obtain non asymptotic results.

4.13. Proposition. Assume that $\mathbf{d}$ is balanced and $\mathbf{m}_{\mathbf{d}} \leq 2 \pi$. Then $\mathbf{m}_{\mathbf{d}, \varepsilon}$ is attained for every $\varepsilon$.

4.14. Remark. When $\mathbf{m}_{\mathbf{d}}<2 \pi, \Omega$ is thin. On the other hand, if $\mathbf{m}_{\mathbf{d}}=2 \pi$, then $\Omega$ is either thin or critical. An example of critical couple $(\Omega, \mathbf{d})$ ( $c f$ Proposition 4.2): a doubly connected domain $\Omega$ of conformal ratio $e^{2}$, and $\mathbf{d}=(1,1)$. For this special case, Proposition 4.13 has been proved in [7, Theorem 1]. More generally, we could consider a balanced couple $(\Omega, \mathbf{d})$ such that $\mathbf{m}_{\mathbf{d}}=2 \pi$ and, for some $j \geq 1, d_{0}=d_{j}= \pm 1$ and $d_{l}=0$ if $l \neq j$.

Proof. We start by noting that

(4.23) $\mathbf{m}_{\mathbf{d}, \varepsilon}<\mathbf{m}_{\mathbf{d}}, \quad \forall \varepsilon>0, \forall \mathbf{d} \in \mathscr{B} \backslash\{0\}$.

Indeed, let $v \in \mathscr{E}_{\mathbf{d}}$ attain the minimum of $\mathbf{m}_{\mathbf{d}}$. Consider the minimization problem

(4.24) $\min \left\{E_{\varepsilon}(w) ; \operatorname{tr} w=\operatorname{tr} v\right\}$.

If $u$ is a minimizer in (4.24), then $u \in \mathscr{E}_{\mathbf{d}}$ and $u$ satisfies the equation $-\Delta u=\frac{1}{\varepsilon^{2}} u\left(1-|u|^{2}\right)$ in $\Omega$. If $u=v$, then $|u|=1$, and this implies $u$ constant (since $|\operatorname{tr} u|=1$ ). Thus $\mathbf{d}=0$. Consequently, when $\mathbf{d} \neq 0, v$ is not a minimizer in (4.24), and thus $\mathbf{m}_{\mathbf{d}, \varepsilon}<\mathbf{m}_{\mathbf{d}}$.

In particular, if $\mathbf{m}_{\mathbf{d}} \leq 2 \pi$ then $\mathbf{m}_{\mathbf{d}, \varepsilon}<2 \pi$. Consider a minimizing sequence $\left(u_{n}\right)$ for $E_{\varepsilon}$ in $\mathscr{E}_{\mathbf{d}}$, and assume that $u_{n} \rightarrow u \in \mathscr{E}_{\mathbf{e}}$. By the Price lemma, we find that

$$
2 \pi>\mathbf{m}_{\mathbf{d}, \varepsilon} \geq \frac{1}{2} \int_{\Omega}|\nabla u|^{2}+\pi|\mathbf{d}-\mathbf{e}| .
$$

We prove by contradiction that $\mathbf{e}=\mathbf{d}$. Otherwise, (4.25) implies that $|\mathbf{e}-\mathbf{d}|=1$; in particular $\mathbf{e}$ is unbalanced. Therefore,

$$
\int_{\Omega}|\nabla u|^{2} \geq 2\left|\int_{\Omega} \operatorname{Jac} u\right|=2 \pi\left|d_{0}-\sum_{j \geq 1} d_{j}\right| \geq 2 \pi .
$$

We obtain a contradiction by combining (4.25) with (4.26).

We next turn to existence of minimizers in thick domains. We conjecture that minimizers do not exist for small $\varepsilon$. We present below a partial result supporting this conjecture. 
4.15. Definition. Let $\Omega$ be a domain with associated degrees $\mathbf{d} \neq 0$. Consider the minimization problem

(4.27) $\min \left\{\mathbf{m}_{\mathbf{e}}+\pi|\mathbf{d}-\mathbf{e}| ; \mathbf{e} \in \mathscr{B}\right\}$.

$\Omega$ is fat provided $\mathbf{e}=0$ is the unique solution of (4.27).

Equivalently, $\Omega$ is fat provided $\mathbf{d} \neq 0$ and

(4.28) $\mathbf{m}_{\mathbf{e}}+\pi|\mathbf{d}-\mathbf{e}|>\pi|\mathbf{d}|, \quad \forall \mathbf{e} \in \mathscr{B} \backslash\{0\}$.

Note that a fat domain is automatically thick.

Let us first give some examples. A simply connected domain is always fat.

Consider next a doubly connected domain $\Omega$ with balanced degrees $\mathbf{d}=(d, d) \neq 0$. Using Proposition 4.2 , it is easy to see that $\Omega$ is fat if and only if its conformal ratio is $>e^{2}$. By Proposition 4.8 , when $|d|=1$ fat is the same as thick. However, when $|d| \geq 2$, there is a gap between fat and thick domains.

We consider next some examples of unbalanced degrees in doubly connected domains. If $\mathbf{d}=$ $\left(d_{1}, d_{2}\right)$ and $d_{1} d_{2} \leq 0$, then $\Omega$ is always fat. If $d_{1} d_{2}>0$, then $\Omega$ fat amounts to $R>e^{2 / \min \left(\left|d_{1}\right|,\left|d_{2}\right|\right)}$.

A final example in triply connected domains. Consider $\Omega_{t}$ as in Fig. 2. As in the proof of Proposition 4.10, we have $\mathbf{m}_{\mathbf{e}} \rightarrow \infty$ as $t \backslash 0$, for each balanced collection $\mathbf{e} \neq 0$. We find that, for each $\mathbf{d} \neq 0, \Omega_{t}$ is fat for small $t$.

The main nonexistence result for semi-stiff Ginzburg-Landau minimizers was obtained by Berlyand, Golovaty and Rybalko [5]. Our next result is a slight generalization of the main result in[5], and the proof follows very closely the one in [5].

4.16. Theorem. Assume that $\Omega$ is fat. Then, for small $\varepsilon$, the infimum $\mathbf{m}_{\mathbf{d}, \varepsilon}$ is not attained.

Proof. We argue by contradiction and assume that, for a sequence $\varepsilon_{n} \rightarrow 0$, there exists $u_{\varepsilon_{n}}$ minimizer of $E_{\varepsilon_{n}}$ in $\mathscr{E}_{\mathbf{d}}$. In order to keep notation simple, we omit the subscript $n$.

Step 1. Identification of the limit of $u_{\varepsilon}$.

Using the upper bound (4.21), we find that, possibly up to a subsequence, $u_{\varepsilon} \rightarrow u$ to some circlevalued limit $u \in \mathscr{E}_{\mathbf{e}}$. By Proposition 2.3, e is balanced. By applying the Price lemma and using Proposition 4.3, we find that

$$
\begin{aligned}
\mathbf{m}_{\mathbf{e}}+\pi|\mathbf{d}-\mathbf{e}|+o(1) & \leq \frac{1}{2} \int_{\Omega}|\nabla u|^{2}+\pi|\mathbf{d}-\mathbf{e}|+o(1) \leq \liminf _{\varepsilon \rightarrow 0} E_{\varepsilon}\left(u_{\varepsilon}\right) \\
& \leq \liminf _{\varepsilon \rightarrow 0} \mathbf{m}_{\mathbf{f}, \varepsilon}+\pi|\mathbf{d}-\mathbf{f}| \leq \mathbf{m}_{\mathbf{f}}+\pi|\mathbf{d}-\mathbf{f}|, \quad \forall \mathbf{f} \in \mathscr{B} .
\end{aligned}
$$

This implies that $\mathbf{e}=0$ and thus $u$ is a constant of modulus 1 . With no loss of generality, we assume that $u=1$.

Step 2. Exponential localization of $u_{\varepsilon}$ near $\partial \Omega$.

The idea is to combine the upper bound $\mathbf{m}_{\mathbf{d}, \varepsilon} \leq \pi|\mathbf{d}|$ (provided by (4.4)) with an opposite inequality obtained by integrating the inequality $|\nabla u|^{2} \geq 2 \mid$ Jac $u \mid$.

Let, for $t>0, \Omega_{t}=\{x \in \Omega ; \operatorname{dist}(x, \partial \Omega)>t\}$, and $\Gamma_{j, t}=\left\{x \in \Omega ; \operatorname{dist}\left(x, \Gamma_{j}\right)=t\right\}$. The starting point is the fact that $\left|u_{\varepsilon}\right| \geq \frac{1}{2}$ in $\Omega_{t}$ provided $t \geq C \varepsilon$. This follows from the upper bound $E_{\varepsilon}\left(u_{\varepsilon}\right) \leq \pi|\mathbf{d}|$ combined with the Ginzburg-Landau equation satisfied by $u_{\varepsilon}$; see [29]. The same ingredients imply that $u_{\varepsilon} \rightarrow u$ in $C_{l o c}^{\infty}(\Omega)$ as $\varepsilon \rightarrow 0$ [29]. This convergence combined with the homotopical invariance of the degree imply that, for small $\varepsilon$ and sufficiently small $\delta$, we have

$$
\operatorname{deg}\left(u_{\varepsilon}, \Gamma_{j, t}\right)=0 \quad C \varepsilon \leq t \leq \delta .
$$

In particular, in $\Omega_{C \varepsilon}$ we may write $u_{\varepsilon}=\rho_{\varepsilon} e^{\imath \varphi_{\varepsilon}}$, where $\rho_{\varepsilon}=\left|u_{\varepsilon}\right| \in[1 / 2,1]$.

Consider now the function

$$
f_{\varepsilon}(t)=\frac{1}{2} \int_{\Omega_{t}}\left|\nabla u_{\varepsilon}\right|^{2}+\frac{1}{4 \varepsilon^{2}} \int_{\Omega_{t}}\left(1-\left|u_{\varepsilon}\right|^{2}\right)^{2}
$$


By integrating over $\Omega \backslash \Omega_{t}$ the inequality $\left|\nabla u_{\varepsilon}\right|^{2} \geq 2\left|\operatorname{Jac} u_{\varepsilon}\right|$, we find that

$$
\begin{aligned}
\frac{1}{2} \int_{\Omega \backslash \Omega_{t}}\left|\nabla u_{\varepsilon}\right|^{2} & \geq \frac{1}{2} \sum_{j}\left|\int_{\Gamma_{j}} u_{\varepsilon} \wedge \frac{\partial u_{\varepsilon}}{\partial \tau}-\int_{\Gamma_{j, t}} u_{\varepsilon} \wedge \frac{\partial u_{\varepsilon}}{\partial \tau}\right|=\frac{1}{2} \sum_{j}\left|2 \pi d_{j}-\int_{\Gamma_{j, t}} u_{\varepsilon} \wedge \frac{\partial u_{\varepsilon}}{\partial \tau}\right| \\
& \geq \pi|\mathbf{d}|-\frac{1}{2} \sum_{j}\left|\int_{\Gamma_{j, t}} u_{\varepsilon} \wedge \frac{\partial u_{\varepsilon}}{\partial \tau}\right|=\pi|\mathbf{d}|-\frac{1}{2} \sum_{j}\left|\int_{\Gamma_{j, t}} \rho_{\varepsilon}^{2} \frac{\partial \varphi_{\varepsilon}}{\partial \tau}\right| \\
& =\pi|\mathbf{d}|-\frac{1}{2} \sum_{j}\left|\int_{\Gamma_{j, t}}\left(\rho_{\varepsilon}^{2}-1\right) \frac{\partial \varphi_{\varepsilon}}{\partial \tau}\right| .
\end{aligned}
$$

On the one hand, we have

$$
\begin{aligned}
\sum_{j}\left|\int_{\Gamma_{j, t}}\left(\rho_{\varepsilon}^{2}-1\right) \frac{\partial \varphi_{\varepsilon}}{\partial \tau}\right| & \leq C \varepsilon \int_{\Gamma_{j, t}}\left(\left|\nabla \varphi_{\varepsilon}\right|^{2}+\frac{1}{\varepsilon^{2}}\left(1-\rho_{\varepsilon}^{2}\right)^{2}\right) \\
& \leq C \varepsilon \int_{\Gamma_{j, t}}\left(\frac{1}{2}\left|\nabla u_{\varepsilon}\right|^{2}+\frac{1}{4 \varepsilon^{2}}\left(1-\left|u_{\varepsilon}\right|^{2}\right)^{2}\right) .
\end{aligned}
$$

On the other hand, the coarea formula implies that (for $t<\delta$ sufficiently small) we have

$$
f_{\varepsilon}^{\prime}(t)=-\sum_{j} \int_{\Gamma_{j, t}}\left(\frac{1}{2}\left|\nabla u_{\varepsilon}\right|^{2}+\frac{1}{4 \varepsilon^{2}}\left(1-\left|u_{\varepsilon}\right|^{2}\right)^{2}\right) .
$$

If we combine (4.32)-(4.34) with the upper bound $\mathbf{m}_{\mathbf{d}, \varepsilon} \leq \pi|\mathbf{d}|$, we find that

$$
C_{1} \varepsilon f_{\varepsilon}^{\prime}(t)+f_{\varepsilon}(t)+\frac{1}{\varepsilon^{2}} \int_{\Omega \backslash \Omega_{t}}\left(1-\left|u_{\varepsilon}\right|^{2}\right)^{2} \leq 0 \quad \forall C \varepsilon \leq t \leq \delta .
$$

By integrating this inequality, we find that

$$
\int_{\Omega_{t}}\left|\nabla u_{\varepsilon}\right|^{2} \leq C_{2} e^{-t /\left(C_{1} \varepsilon\right)}, \forall C \varepsilon \leq t \leq \delta, \text { and } \frac{1}{\varepsilon^{2}} \int_{\Omega}\left(1-\left|u_{\varepsilon}\right|^{2}\right)^{2} \leq C_{2} e^{-C_{3} / \varepsilon},
$$

that is the potential part is exponentially small and energy is exponentially small outside a thin layer around $\partial \Omega$.

For further use, let us note that by combining (4.32) with (4.36) and with a mean value argument, we obtain the following

(4.37) $E_{\varepsilon}\left(u_{\varepsilon}\right) \geq \pi|\mathbf{d}|-C_{4} e^{-C_{5} / \varepsilon}$.

Step 3. Pointwise decay of $\nabla u_{\varepsilon}$ far away from $\partial \Omega$.

The starting point is the following quantitative result [9, Lemma 22].

4.17. Lemma. Let $u$ be a critical point of $E_{\varepsilon}$ in $\mathbb{D}_{R}$ satisfying:

1. $R \geq \varepsilon$.

2. $E_{\varepsilon}(u)=K^{2} \leq \frac{\varepsilon}{R}$.

3. $\frac{1}{2} \leq\left|u_{\varepsilon}\right| \leq 1$.

Then

(4.38) $|\nabla u(0)| \leq C \frac{K}{R}$ and $1-|u(0)|^{2} \leq C \frac{K^{2} \varepsilon^{2}}{R^{2}}$.

Using Lemma 4.17, we easily obtain the existence of $a=a_{\varepsilon} \in \mathbb{R}$ such that, with $z_{\varepsilon}:=e^{\imath a_{\varepsilon}}$, we have, for fixed $m \in \mathbb{N}^{*}$ and $\delta>0$ and sufficiently small $\varepsilon$,

(4.39) $\left|\nabla u_{\varepsilon}\right| \leq \varepsilon^{m},\left|\varphi_{\varepsilon}-a_{\varepsilon}\right| \leq \varepsilon^{m},\left|u_{\varepsilon}-z_{\varepsilon}\right| \leq \varepsilon^{m} \quad$ in $\Omega_{\delta}$.

Step 4. Idea of [5]: reduction to a linear problem.

To start with, assume that one of the components of $\partial \Omega$ is a circle, say $\Gamma_{0}=C(0, R) .{ }^{11}$ If we multiply

\footnotetext{
${ }^{11} \mathrm{As}$ we will see later, this is not relevant for the analysis.
} 
by $\ln \frac{|x|}{R}$ the equation satisfied by $u_{\varepsilon}$ and integrate over $\mathbb{D}_{R} \backslash \mathbb{D}_{R-\delta}$, we find that

$$
\frac{1}{R} \int_{C(0, R)} u_{\varepsilon}=\frac{1}{R-\delta} \int_{C(0, R-\delta)} u_{\varepsilon}+\ln \frac{R-\delta}{R} \int_{C(0, R-\delta)} \frac{\partial u_{\varepsilon}}{\partial v}+\frac{1}{\varepsilon^{2}} \int_{\mathbb{D}_{R} \backslash \mathbb{D}_{R-\delta}} \ln \frac{|x|}{R} u_{\varepsilon}\left(1-\left|u_{\varepsilon}\right|^{2}\right)
$$

By combining (4.40) with (4.36) and (4.39), we find that, possibly after multiplying $u_{\varepsilon}$ with a suitable constant of modulus 1 , the following hold:

$$
\int_{C(0, R)} u_{\varepsilon} \in \mathbb{R}_{+},\left|\int_{C(0, R)} u_{\varepsilon}-1\right| \leq \varepsilon^{m} \text { and }\left|u_{\varepsilon}-1\right| \leq \varepsilon^{m} \quad \text { in } \Omega_{\delta}
$$

Using the last estimate in (4.41) for $m=5$ together with the straightforward inequality

$$
\left(1-|z|^{2}\right)^{2} \geq(\operatorname{Re} z-1)^{2}(\operatorname{Re} z+1)^{2}-4(1-\operatorname{Re} z)^{2}(\operatorname{Im} z)^{2}, \quad \forall|z| \leq 1,
$$

we find, for fixed small $\delta>0$ and small $\varepsilon>0$ :

$$
\begin{aligned}
E_{\varepsilon}\left(u_{\varepsilon}, \mathbb{D}_{R} \backslash \mathbb{D}_{R-2 \delta}\right) & =\frac{1}{2} \int_{\mathbb{D}_{R} \backslash \mathbb{D}_{R-2 \delta}}\left(\left|\nabla u_{\varepsilon}\right|^{2}+\frac{1}{2 \varepsilon^{2}}\left(1-\left|u_{\varepsilon}\right|^{2}\right)^{2}\right) \geq F_{\varepsilon, \delta}\left(u_{\varepsilon}\right) \\
& :=\frac{1}{2} \int_{\mathbb{D}_{R} \backslash \mathbb{D}_{R-2 \delta}}\left|\nabla u_{\varepsilon}\right|^{2}+\frac{1}{2} \int_{\mathbb{D}_{R-\delta} \backslash \mathbb{D}_{R-2 \delta}}\left[\varepsilon^{-2}\left(\operatorname{Re} u_{\varepsilon}-1\right)^{2}-\varepsilon^{2}(\operatorname{Im} w)^{2}\right] .
\end{aligned}
$$

To summarize: assuming $\Gamma_{0}$ a round circle, we found that

$$
E_{\varepsilon}\left(u_{\varepsilon}, \mathbb{D}_{R} \backslash \mathbb{D}_{R-2 \delta}\right) \geq M_{\varepsilon, \delta, R, d_{0}}\left(g_{0}\right)
$$

where $g_{0}=g_{0, \varepsilon}=u_{\varepsilon \mid C(0, R)}$ satisfies $\operatorname{deg}\left(g_{0}, C(0, R)\right)=d_{0}$ and $\int_{C(0, R)} g_{0} \geq 0$ and

$$
M_{\varepsilon, \delta, R}\left(g_{0}\right)=\min \left\{F_{\varepsilon, \delta}(v) ; \operatorname{tr} v=g_{0}\right\} .
$$

We next claim that, without any assumption on the geometry of $\Gamma_{j}$, we can find appropriate constants $C_{j}, R_{j}>0$ and $g_{j}$ such that

$$
E_{\varepsilon}\left(u_{\varepsilon},\left\{x \in \Omega ; \operatorname{dist}\left(x, \Gamma_{j}\right)<\delta\right\}\right) \geq M_{\varepsilon, C_{j} \delta, R_{j}}\left(g_{j}\right)
$$

here, $g_{j}=g_{j, \varepsilon}$ satisfies $\operatorname{deg}\left(g_{j}, C\left(0, R_{j}\right)\right)=d_{j}$ and $\int_{C\left(0, R_{j}\right)} g_{j} \geq 0$. This is achieved via a conformal transform mapping $\Gamma_{j}$ onto a circle $C\left(0, R_{j}\right)$ and $\Omega$ into a subset of $\mathbb{D}$. Such a transform affects $E_{\varepsilon}$ only by a bounded weight in front of $\left(1-\left|u_{\varepsilon}\right|^{2}\right)^{2}$ and, it is clear that the computation leading to (4.41)-(4.43) is still valid. Conclusion of this step: we may find positive numbers $C_{j}, R_{j}, j \in \llbracket 0, k \rrbracket$, and a small $\delta>0$ such that

$$
E_{\varepsilon}\left(u_{\varepsilon}\right) \geq \sum_{j=0}^{k} M_{\varepsilon, C_{j} \delta, R_{j}}\left(g_{j}\right) \text { for small } \varepsilon
$$

Step 5. Conclusion.

We assume e.g. that each $R_{j}$ is 1 , but this is not relevant for the analysis. The minimization of $F_{\varepsilon, \delta}$ with given boundary datum $g=v_{\mid \mathbb{S}^{1}}: \mathbb{S}^{1} \rightarrow \mathbb{S}^{1}$ is a linear problem, and it is possible to compute the minimal energy in function of the Fourier coefficients of $g$; this computation was performed by Berlyand, Golovaty and Rybalko [6]. More precisely, if $g=\sum_{n \geq 0} a_{n} \cos (n \theta)+\sum_{n \geq 1} b_{n} \sin (n \theta)$, then the last condition in (4.44) implies that $a_{0} \in \mathbb{R}_{+}$, and the minimal energy is

$$
P_{0}+\frac{\pi}{2} \sum_{n \geq 1}\left[n P_{n}\left|\operatorname{Re} a_{n}\right|^{2}+n P_{n}\left|\operatorname{Re} b_{n}\right|^{2}+n Q_{n}\left|\operatorname{Im} a_{n}\right|^{2}+n Q_{n}\left|\operatorname{Im} b_{n}\right|^{2}\right]
$$

where the coefficients $P_{n}=P_{n}(\varepsilon, \delta)$ and $Q_{n}=Q_{n}(\varepsilon, \delta)$ are non negative and explicit. Now comes the key argument [5]: for fixed $\delta$ and sufficiently small $\varepsilon$, we have

(4.48) $P_{n} Q_{n}>1$ when $n \geq 1$.

The proof of this inequality is sketched in [6] and explained in detail in [9, Section 4, Step 2]. 
We continue as follows: using (4.47), (4.48) and the degree formula [20]

(4.49) $\operatorname{deg}\left(g, \mathbb{S}^{1}\right)=\sum_{n \geq 1} n\left(\operatorname{Re} a_{n} \operatorname{Im} b_{n}-\operatorname{Re} b_{n} \operatorname{Im} a_{n}\right)$

(which is nothing else than a rewriting of (2.1)), we find that

$$
M_{\varepsilon, C_{j} \delta_{j}, R_{j}}\left(g_{j}\right) \geq \pi \sum_{n \geq 1} n \sqrt{P_{n} Q_{n}}\left(\left|\operatorname{Re} a_{n}\right|\left|\operatorname{Im} b_{n}\right|+\left|\operatorname{Re} b_{n}\right|\left|\operatorname{Im} a_{n}\right|\right) \geq \pi\left|d_{j}\right|,
$$

the last equality being strict when $d_{j} \neq 0$. By combining this with (4.46), we find that

$$
\mathbf{m}_{\mathbf{d}, \varepsilon}=E_{\varepsilon}\left(u_{\varepsilon}\right)>\pi|\mathbf{d}| \text { for small } \varepsilon
$$

which contradicts the upper bound $\mathbf{m}_{\mathbf{d}, \varepsilon} \leq \pi|\mathbf{d}|(c f(4.4))$.

Concerning the attainability of $\mathbf{m}_{\mathbf{d}, \varepsilon}$ in critical domains, we already know from Proposition 4.13 that $\mathbf{m}_{\mathbf{d}, \varepsilon}$ is attained if $\mathbf{m}_{\mathbf{d}}=2 \pi$. In Section 8 , we will speculate about what happens in the remaining cases.

\section{CRITICAL POINTS IN MULTiPly CONNECTED DOMAINS}

Let us start with a simple result. Let $\mathbf{d}$ be a balanced configuration. Since $\mathbf{d}$ is balanced, the class

$$
\mathscr{F}=\left\{u \in \mathscr{E}_{\mathbf{d}} ; 1 / 2 \leq|u| \leq 2\right\}
$$

is non empty. We may thus consider the minimization problem

$$
\mathbf{m}_{\mathbf{d}, \varepsilon}^{*}=\min \left\{E_{\varepsilon}(u) ; u \in \mathscr{F}\right\}
$$

The next result is reminiscent from the existence of permanent currents in 3D tori [31]; it has been established in [8] in a special case.

5.1. Proposition. For small $\varepsilon, \mathbf{m}_{\mathbf{d}, \varepsilon}^{*}$ is attained by some $u_{\varepsilon}$ which is a stable critical point of $E_{\varepsilon}$ in $\mathscr{E}_{\mathbf{d}}$

Sketch of proof. Using the upper bound $E_{\varepsilon}\left(u_{\varepsilon}\right) \leq \mathbf{m}_{\mathbf{d}}$, it is easy to see that, possibly after multiplying $u_{\varepsilon}$ by a suitable complex number of modulus 1 , we have $u_{\varepsilon} \rightarrow u$ in $H^{1}(\Omega)$, where $u$ is a minimizer of $\mathbf{m}_{\mathbf{d}}$ in $\mathscr{F}_{\mathbf{d}}$. Let $g=u_{\mid \partial \Omega}$ and $g_{\varepsilon}=u_{\varepsilon \mid \partial \Omega}$. Then

$$
g_{\varepsilon} \rightarrow g \text { in } H^{1 / 2}(\partial \Omega) \text {. }
$$

We next rely on the following [8, Lemma 12.1].

5.2. Lemma. If (5.3) holds and if $v_{\varepsilon}$ minimizes $E_{\varepsilon}$ under the constraint $\operatorname{tr} v_{\varepsilon}=g_{\varepsilon}$, then $\left|v_{\varepsilon}\right| \rightarrow 1$ uniformly in $\Omega$ as $\varepsilon \rightarrow 0$.

Using the above lemma, we find that, for small $\varepsilon, u_{\varepsilon}$ is necessarily one of the minimizers of $E_{\varepsilon}$ under the constraint $\operatorname{tr} u_{\varepsilon}=g$. In particular, $u_{\varepsilon}$ satisfies $-\Delta u_{\varepsilon}=\frac{1}{\varepsilon^{2}} u_{\varepsilon}\left(1-\left|u_{\varepsilon}\right|^{2}\right)$. The validity of the third condition in (2.5) is obtained via the fact that

$$
E_{\varepsilon}\left(u_{\varepsilon}\right) \leq E_{\varepsilon}\left(u_{\varepsilon} e^{\imath \psi}\right), \quad \forall \psi \in H^{1}(\Omega) .
$$

Thus $u_{\varepsilon}$ is a critical point of $E_{\varepsilon}$ in $\mathscr{E}_{\mathbf{d}}$.

There are several ways to obtain the stability of $u_{\varepsilon}$. A possibility is to note that, since $\left|u_{\varepsilon}\right| \rightarrow 1$, $u_{\varepsilon}$ is a minimizer of $E_{\varepsilon}$ is a small $C^{1}$-neighborhood of $u_{\varepsilon}$ ( $c f$ the definition of the class $\left.\mathscr{F}\right)$. Then we invoke the " $H^{1}$ versus $C^{1}$ minimizers" result of Brezis and Nirenberg [19]. However, this requires adapting the arguments in [19], since the result there is about scalar problems.

Alternatively, arguing by contradiction, we may find (possibly along some sequence) some $w_{\varepsilon}$ such that $\left\|w_{\varepsilon}-u_{\varepsilon}\right\|_{H^{1}(\Omega)} \rightarrow 0$ and $E_{\varepsilon}\left(w_{\varepsilon}\right)<E_{\varepsilon}\left(u_{\varepsilon}\right)$. Arguing as above, we have $\operatorname{tr} w_{\varepsilon} \rightarrow g$ in $H^{1 / 2}(\partial \Omega)$, and thus for small $\varepsilon$, the minimizer $y_{\varepsilon}$ of $E_{\varepsilon}$ with the constraint $\operatorname{tr} y_{\varepsilon}=\operatorname{tr} w_{\varepsilon}$ lies in $\mathscr{F}$. This yields the contradiction $E_{\varepsilon}\left(u_{\varepsilon}\right) \leq E_{\varepsilon}\left(y_{\varepsilon}\right) \leq E_{\varepsilon}\left(w_{\varepsilon}\right)<E_{\varepsilon}\left(u_{\varepsilon}\right)$. 
The case of unbalanced degrees is much more involved. In a beautiful paper [11], Berlyand and Rybalko obtained existence of critical points of $E_{\varepsilon}$ in $\mathscr{E}_{\mathbf{d}}$ provided $\varepsilon$ is small and $\Omega$ is doubly connected. This second condition is crucial in their approach, and can be seen as an avatar of the work of Bahri and Coron [4] on the influence of the topology of the domains on the existence of non trivial solutions of the critical equation $-\Delta u=u^{(n+2) /(n-2)}$. Later, Dos Santos [22] extended the result in [11] to general multiply connected domains, and simplified the proofs in [11]. Their combined results give the following

5.3. Theorem. Let $\Omega$ be multiply connected and let $M \in \mathbb{N}^{*}$. Let $\mathbf{d}$ be arbitrary. Then, for small $\varepsilon$, $E_{\varepsilon}$ has at least $M$ stable critical points in $\mathscr{E}_{\mathbf{d}}$.

Though we are not going to detail their arguments here, we will explain part of their approach on an example.

Main idea of the proof (cf $\left[11\right.$, Section 5]). Assume that $\Omega$ is doubly connected, say $\Omega=\mathbb{D} \backslash \overline{\mathbb{D}_{R}}$, and that $\mathbf{d}=(1,0)$. Note that, in this case, we know that $\mathbf{m}_{\mathbf{d}, \varepsilon}$ is not attained (Proposition 3.3). Let, for small $\varepsilon, u_{\varepsilon}$ be the minimizer of $\mathbf{m}_{\mathbf{d}, \varepsilon}^{*}$ constructed in Proposition 5.1. By a very involved construction of test functions, Berlyand and Rybalko obtained the following

5.4. Lemma. Fix an arbitrary neighborhood $\omega$ of $\Gamma_{0}=\mathbb{S}^{1}$. Then there is some $v_{\varepsilon} \in \mathscr{E}_{\mathbf{d}}$ such that $\left|v_{\varepsilon}\right| \leq 1, v_{\varepsilon}=u_{\varepsilon}$ outside $\omega$, and

$$
E_{\varepsilon}\left(v_{\varepsilon}\right)<E_{\varepsilon}\left(u_{\varepsilon}\right)+\pi
$$

Let us emphasize the fact that it is easy to construct $v_{\varepsilon} \in \mathscr{E}_{\mathbf{d}}$ such that $E_{\varepsilon}\left(v_{\varepsilon}\right)=E_{\varepsilon}\left(u_{\varepsilon}\right)+\pi+o(1)$ : for this purpose, it suffices to consider a product of the form $u_{\varepsilon} M_{a}$ (with $M_{a}$ a Moebius transform), then modify it on $\partial \Omega$ in order to obtain a map in $\mathscr{E}_{\mathbf{d}}$ and then let $a \rightarrow \mathbb{S}^{1}$. The key fact in the above is the strict inequality in (5.4). Though the main idea consists in considering a test function mimicking the product $u_{\varepsilon} M_{a}$, the heart of the proof consists in finding the sign of the difference $E_{\varepsilon}\left(v_{\varepsilon}\right)-E_{\varepsilon}\left(u_{\varepsilon}\right)-\pi$; the fact that this sign is minus relies on the fact that the boundary degrees of $u_{\varepsilon}$ are above the ones of $v_{\varepsilon}$. For example, a similar result allows to go from degrees 2 and 2 for $u_{\varepsilon}$ to degrees 2 and 1 (or 2 and 0 ) for $v_{\varepsilon}$, but it seems that it is not possible to increase the degrees by such a construction.

A second ingredient is the "approximate degree", reminiscent of a similar notion introduced by Almeida [1]. If $u$ is circle-valued in $\Omega$, then we have in polar coordinates

$$
d:=\operatorname{deg}(u, C(0, R))=\operatorname{deg}\left(u, \mathbb{S}^{1}\right)=\frac{1}{2 \pi(R-1)} \int_{\Omega} \frac{1}{r} u \wedge \frac{\partial u}{\partial \theta} .
$$

Let now $u$ be arbitrary and let $F(u)$ be the the right-hand side of (5.5). Then $F$ is clearly weakly continuous. The following result is not too difficult; for related results, see [11, Lemma 19, Proposition 11].

5.5. Lemma. Let $w_{\varepsilon} \in \mathscr{E}_{\mathbf{d}}$ satisfy $E_{\varepsilon}\left(w_{\varepsilon}\right) \leq C$. Then

1. We have

$$
\lim _{\varepsilon \rightarrow 0} \operatorname{dist}\left(F\left(w_{\varepsilon}\right), \mathbb{Z}\right)=0 .
$$

In particular, for fixed $C$ and small $\varepsilon$ the classes

$$
\mathscr{G}_{\mathbf{d}, d}=\left\{u \in \mathscr{E}_{\mathbf{d}} ; E_{\varepsilon}(u) \leq C|,| F(u)-d \mid<1 / 2\right\}, \quad d \in \mathbb{Z},
$$

are the (relatively) open connected components of the set $\left\{u \in \mathscr{E}_{\mathbf{d}} ; E_{\varepsilon}(u) \leq C\right\}$.

2. (Generalization of the Price lemma) If $w_{\varepsilon} \in \mathscr{E}_{\mathbf{e}}, E_{\varepsilon}\left(w_{\varepsilon}\right) \leq C$ and $F\left(w_{\varepsilon}\right) \rightarrow d \in \mathbb{Z}$, then

$$
\liminf _{\varepsilon \rightarrow 0} \frac{1}{2} \int_{\Omega}\left|\nabla w_{\varepsilon}\right|^{2} \geq \mathbf{m}_{(d, d)}+\pi|\mathbf{d}-\mathbf{e}| .
$$

3. (Generalization of the Proposition 5.1) Let $d \in \mathbb{Z}$ and set $\mathbf{f}=(d, d)$. Then, for small $\varepsilon$, the minimizers of $E_{\varepsilon}$ in $\mathscr{G}_{\mathbf{f}, d}$ are precisely the minimizers of $\mathbf{m}_{\mathbf{f}, \varepsilon}^{*}$. 
Finally, existence of a stable critical point of $E \varepsilon$ in $\mathscr{E}_{\mathbf{d}}$ is obtained as follows: consider the minimization problem

$$
\widetilde{\mathbf{m}}_{\mathbf{d}, \varepsilon}:=\inf \left\{E_{\varepsilon}(u) ; u \in \mathscr{E}_{\mathbf{d}},|F(u)-1|<1 / 2\right\}
$$

Assume for the moment that, in the above, inf is actually min. By Lemma 5.5, for small $\varepsilon$ the minimum is attained by some $w_{\varepsilon}$ such that $\left|F\left(w_{\varepsilon}\right)-1\right|<1 / 3$. By continuity of $F, w_{\varepsilon}$ is a local minimizer of $E_{\varepsilon}$, and thus $w_{\varepsilon}$ satisfies (2.4).

It remains to prove that inf is attained in (5.8). Consider a minimizing sequence $\left(y_{j}\right)$ for $\widetilde{\mathbf{m}}_{\mathbf{d}, \varepsilon}$ such that $y_{j} \rightarrow y$ for some $y=y_{\varepsilon}$. Possibly after passing to a sequence $\varepsilon_{n} \rightarrow 0$, we may assume that $y \in \mathscr{E}_{\mathbf{e}}$ for a fixed e. Invoking the fact that clearly the map $v_{\varepsilon}$ in Lemma 5.4 belongs to $\mathscr{G}_{\mathbf{d}, d}$ for small $\varepsilon$, together with the Price lemma, Lemma 5.4 and (5.7), we find that:

$$
\begin{aligned}
\mathbf{m}_{(1,1)}+\pi(|\mathbf{d}-\mathbf{e}|+|\mathbf{e}-(1,1)|)+o(1) & \leq E_{\varepsilon}(y)+\pi|\mathbf{d}-\mathbf{e}| \leq \widetilde{\mathbf{m}}_{\mathbf{d}, \varepsilon}<E_{\varepsilon}\left(u_{\varepsilon}\right)+\pi \\
& =\mathbf{m}_{(1,1), \varepsilon}^{*}+\pi \leq \mathbf{m}_{(1,1)}+\pi .
\end{aligned}
$$

This leaves the options $\mathbf{e}=(1,0)$ (which is what we want) and $\mathbf{e}=(1,1)$. Argue by contradiction and assume that $\mathbf{e}=(1,1)$. Then Lemma 5.53 implies that $E_{\varepsilon}(y)=\mathbf{m}_{(1,1), \varepsilon}^{*}$. We obtain a contradiction via (5.9).

The above yields existence of a stable critical point when $\mathbf{d}=(1,0)$.

An adaptation of the above argument implies that, given $\mathbf{e}=(e, e)$ balanced such that $e \geq d_{j}$, $j=0,1$, it is possible to construct, for small $\varepsilon$, critical points $y$ of $E_{\varepsilon}$ in $\mathscr{E}_{\mathbf{d}}$ such that $|F(y)-e|<1 / 2$.

The case of a multiply connected domain follows similar lines.

\section{CRITICAL POINTS IN SIMPLY CONNECTED DOMAINS}

In a simply connected domain, we identify d with the integer $d_{0}$, and write $\mathscr{E}_{d_{0}}$ instead of $\mathscr{E}_{\mathbf{d}}$. The main result in this section is the following

6.1. Theorem ([10]). Let $\Omega$ be simply connected. Then, for large $\varepsilon, E_{\varepsilon}$ has critical points in $\mathscr{E}_{1}$.

Note that it is not possible to reproduce the approach in the previous section: there are no balanced collections to start with. Instead, we rely on a minimax approach that we describe below.

To start with, after a conformal change of variables, we may assume that $\Omega=\mathbb{D}$ and

$$
E_{\varepsilon}(u)=\frac{1}{2} \int_{\mathbb{D}}|\nabla u|^{2}+\int_{\mathbb{D}} \frac{w}{4 \varepsilon^{2}}\left(1-|u|^{2}\right)^{2} ;
$$

here $w \in C^{\infty}\left(\overline{\mathbb{D}} ;(0, \infty)\right.$ is the Jacobian of the conformal transform. For large $\varepsilon$, the energy $E_{\varepsilon}$ is strictly convex, and thus we may associate to each $g \in H^{1 / 2}\left(\mathbb{S}^{1} ; \mathbb{C}\right)$ the minimizer $u=T(g)$ of $E_{\varepsilon}$ subject to $\operatorname{tr} u=g$. We introduce the following notations:

$$
X=\left\{g \in H^{1 / 2}\left(\mathbb{S}^{1} ; \mathbb{S}^{1}\right) ; \operatorname{deg}\left(g, \mathbb{S}^{1}\right)=1\right\},
$$

$N_{\alpha, a}=\operatorname{tr} M_{\alpha, a}, N_{a}=\operatorname{tr} M_{a}$ (recall that $M_{\alpha, a}$ are the Moebius transforms).

We let $0<r<1$ and consider, for large $\varepsilon$, the following minimax problem:

$$
m_{r, \varepsilon}=\inf \left\{\max _{\overline{\mathbb{D}_{r}}} E_{\varepsilon}(T(F(a))) ; F \in C\left(\overline{\mathbb{D}_{r}} ; X\right), F(a)=N_{a} \text { for every } a \in C(0, r)\right\} ;
$$

the heart of the proof of Theorem 6.1 consists in proving that, for large $\varepsilon$ and $r$ close to 1 , the infimum is attained in (6.1), and that this leads to the existence of a minimax critical point of $E_{\varepsilon}$ in $\mathscr{E}_{1}$.

We start by presenting the technical ingredients needed in the proof.

A first tool is the description of the maps in $X$ which are "close" to restrictions of Moebius maps. By combining the degree formula (2.1) with the identity $|\nabla u|^{2}=2 \mathrm{Jac} u+4\left|\partial_{\bar{z}} u\right|^{2}$, we find that for each $u \in \mathscr{E}_{1}, g=\operatorname{tr} u$ satisfies

$$
1=\frac{1}{2 \pi} \operatorname{deg}\left(g, \mathbb{S}^{1}\right)=\frac{1}{\pi} \int_{\mathbb{D}} \operatorname{Jac} u=\frac{1}{2 \pi} \int_{\mathbb{D}}|\nabla u|^{2}-\frac{2}{\pi} \int_{\mathbb{D}}\left|\partial_{\bar{z}} u\right|^{2} \leq|g|_{H^{1 / 2}}^{2},
$$


with equality if and only if $u$ is holomorphic. On the other hand, it is standard that holomorphic maps $u$ satisfying $|\operatorname{tr} u|=1$ are precisely the Blaschke products. In conclusion, if $g \in X$, then $|g|_{H^{1 / 2}}=1$ (or $\left.\leq 1\right)$ is equivalent to $g=N_{\alpha, a}$ for some $\alpha \in \mathbb{S}^{1}$ and $a \in \mathbb{D}$. Equivalently,

(6.3) $\min \left\{E_{\infty}(u) ; u \in \mathscr{E}_{1}\right\}$ equals $\pi$ and is attained precisely by the Moebius transforms.

Therefore, the next result describes indeed maps $g \in X$ close (in $H^{1 / 2}$ ) to restrictions to $\mathbb{S}^{1}$ of Moebius transforms.

6.2. Theorem. There exists some $\delta_{0}>0$ and a function $f:\left(0, \delta_{0}\right) \rightarrow(0, \infty)$ such that $\lim _{\delta \rightarrow 0} f(\delta)=0$ with the following property: if $g \in X$ satisfies $|g|_{H^{1 / 2}}^{2}<1+\delta$ for some $\delta<\delta_{0}$, then:

1. The harmonic extension $u=u(g)$ of $g$ has exactly one zero, $a=a(u)=a(g)$.

2. If we write $g=N_{a} e^{\imath \psi}$ with $\psi \in H^{1 / 2}(\Omega ; \mathbb{R})$, then $|\psi|_{H^{1 / 2}} \leq f(\delta)$.

3. The map $g \mapsto a$ is continuous.

4. In addition, given $r \in(0,1)$ and $\mu>0$, we may pick $\delta_{0}$ such that the above hold and $\| \alpha u \circ M_{-a}-$ $I d \|_{C^{2}\left(\mathbb{D}_{r}\right)}<\mu$ for some appropriate $\alpha \in \mathbb{S}^{1}$.

5. In addition to item 4, there exists some $\varepsilon_{0}>0$ such that, for $\varepsilon>\varepsilon_{0}$, the minimizer $v=T(g)$ of $E_{\varepsilon}$ subject to $\operatorname{tr} v=g$ satisfies $\left\|\alpha v \circ M_{-a}-I d\right\|_{C^{2}\left(\mathbb{D}_{r}\right)}<\mu$ for some appropriate $\alpha \in \mathbb{S}^{1}$, and $|v|>1-\mu$ in $\mathbb{D} \backslash \mathbb{D}_{r}$.

6. For $\delta<\delta_{0}$ and $r<1$, the class $\left\{g \in X\right.$; $\left.|g|_{H^{1 / 2}}^{2}<1+\delta,|a(g)| \leq r\right\}$ is weakly sequentially compact.

The proof of Theorem 6.2 is delicate and will be omitted here.

We next translate problem (6.1) into a more tractable one. For this purpose, let $Y=H^{1 / 2}\left(\mathbb{S}^{1} ; \mathbb{R}\right)$ and consider the map $\psi \stackrel{G}{\mapsto} \operatorname{Id} e^{\imath \psi}$, which clearly maps $Y$ onto $X$ (cf Proposition 2.1). It is not difficult to prove the existence of a (unique) continuous map $\mathbb{D} \ni a \mapsto \psi_{a} \in Y$ such that $\psi_{0}=0$ and $N_{a}=G\left(\psi_{a}\right)=\operatorname{Id} e^{l \psi_{a}}$ for each $a \in \mathbb{D}$. Then (6.1) is equivalent to

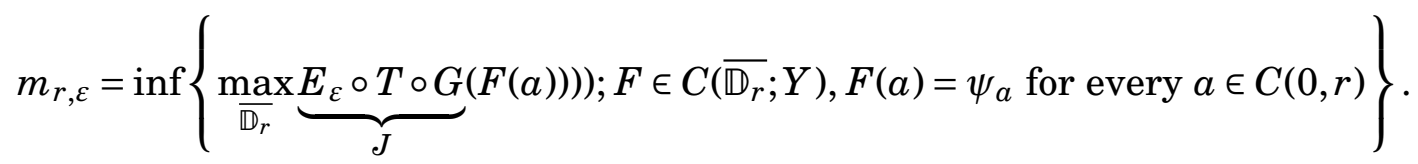

The next result is the following

6.3. Lemma. The map $J=E_{\varepsilon} \circ T \circ G: Y \rightarrow \mathbb{R}$ is $C^{1}$, and we have

$$
J^{\prime}(\psi)(\eta)=\int_{\mathbb{S}^{1}}\left(u \wedge \frac{\partial u}{\partial v}\right) \eta, \quad \forall \psi, \eta \in Y,
$$

where $u=T(G(\psi))$.

Using these ingredients, we may now proceed to the

Proof of Theorem 6.1. Step 1. Mountain pass geometry.

More specifically, we establish the following fact. Let $\varepsilon$ be large and fixed. Then, for $r$ sufficiently close to 1 , we have

$$
m_{r, \varepsilon}>c_{r, \varepsilon}=\max _{C(0, r)} J\left(\psi_{a}\right) .
$$

Indeed, note first that $c_{r, \varepsilon} \rightarrow \pi$ as $r \rightarrow 1$. This is easily obtained by combining the lower bound $c_{r, \varepsilon} \geq \pi$ (cf Proposition 3.3) with the upper bound

$$
c_{r, \varepsilon} \leq \max _{C(0, r)} E_{\varepsilon}\left(M_{a}\right) \rightarrow \pi \quad \text { as } r \rightarrow 1 .
$$

Assume that $m_{r, \varepsilon}<\pi\left(1+\delta_{0}\right)$, with $\delta_{0}$ as in Theorem 6.2 (if this inequality does not hold, then we are done). Let $H$ be the map $g \mapsto a(g)$, defined in Theorem 6.21 , and let $F \in C\left(\overline{\mathbb{D}_{r}} ; Y\right)$ be such that $F(a)=\psi_{a}$ on $C(0, r)$ and $\max _{\overline{\mathbb{D}_{r}}} J \circ F<\pi\left(1+\delta_{0}\right)$. Consider the map $H \circ F: \overline{\mathbb{D}_{r}} \rightarrow \mathbb{D}$. This map is continuous and equals the identity on $C(0, r)$. By the Brouwer fixed point theorem, there is some $a \in \mathbb{D}_{r}$ such that $G(a)=0$. For this $a$, Theorem 6.25 implies that

$$
\frac{1}{\varepsilon^{2}} \int_{\mathbb{D}} w\left(1-|T(F(a))|^{2}\right)^{2} \geq \frac{C}{\varepsilon^{2}},
$$


and thus $m_{r, \varepsilon} \geq \pi+\frac{C}{\varepsilon^{2}}$. We obtain the desired conclusion by letting $r \rightarrow 1$ and noting that $C$ is independent of $r$.

Step 2. Construction of Palais-Smale sequences.

This is straightforward. By Step 1, we are in presence of mountain pass geometry, while, by Lemma 6.3, $J \in C^{1}$. By the Mountain Pass Theorem of Ambrosetti and Rabinowitz [2], we obtain the existence of a sequence $\left(\psi_{j}\right) \subset Y$ such that $J\left(\psi_{j}\right) \rightarrow m_{r, \varepsilon}$ and $J^{\prime}\left(\psi_{j}\right) \rightarrow 0$. Let $g_{j}=G\left(\psi_{j}\right)$ and $u_{j}=T\left(g_{j}\right)$. Let $u$ be such that (up to a subsequence) $u_{j} \rightarrow u$. Clearly, the above, Proposition 2.2 and Lemma 6.3 imply that $u$ is a critical point of $E_{\varepsilon}$, so the remaining issue is to prove that $u \in \mathscr{E}_{1}$.

Step 3. Analysis of the Palais-Smale sequences.

This is the heart of the proof. It is easy to see that $m_{r, \varepsilon} \rightarrow \pi$ as $\varepsilon \rightarrow \infty$. Therefore, for large $\varepsilon$ we have $E_{\varepsilon}\left(u_{j}\right)<\pi\left(1+\delta_{0}\right)$, with $\delta_{0}$ as in Theorem 6.2. By Theorem 6.26 , we have $u \in \mathscr{E}_{1}$ provided that that (up to a subsequence) $\left|H\left(g_{j}\right)\right| \leq r<1$. We prove by contradiction that this holds. ${ }^{12}$ Indeed, assume that $a_{j}:=H\left(g_{j}\right)$ satisfies $\left|a_{j}\right| \rightarrow 1$. We then rescale $u_{j}$ by setting $v_{j}=u_{j} \circ M_{-a_{j}}$. Let $w_{j}=\frac{1}{\varepsilon^{2}} w \circ M_{-a_{j}} \mathrm{Jac} M_{-a_{j}}$, so that $v_{j} \in \mathscr{E}_{1}$ satisfies

$$
\begin{cases}-\Delta v_{j}=w_{j} v_{j}\left(1-\left|v_{j}\right|^{2}\right) & \text { in } \mathbb{D} \\ \left|\int_{\mathbb{D}}\left(v_{j} \wedge \nabla v_{j}\right) \cdot \nabla \zeta\right| \leq o(1)\|\nabla \zeta\|_{L^{2}}, & \forall \zeta \in H^{1}(\mathbb{D}) \\ v_{j}(0) \rightarrow 0 & \end{cases}
$$

and

$$
\frac{1}{2} \int_{\mathbb{D}}\left|\nabla v_{j}\right|^{2}+\frac{1}{4} \int_{\mathbb{D}} w_{j}\left(1-\left|v_{j}\right|^{2}\right)^{2} \rightarrow m_{r, \varepsilon}
$$

Since $\left|a_{j}\right| \rightarrow 1$, we have

$$
w_{j} \rightarrow 0 \text { uniformly on compacts of } \mathbb{D} \text {. }
$$

Using (6.7) together with (6.9), we find that $v_{j} \rightarrow v$, where $v(0)=0$ and $v$ is a critical point of $E_{\infty}$ in $\mathscr{E}_{d}$ for some $d$. For large $\varepsilon$, we have $m_{r, \varepsilon}<2 \pi$, and thus, by the Price lemma, we have

(6.10) $\frac{1}{2} \int_{\mathbb{D}}|\nabla v|^{2}+\pi|d-1|<2 \pi$.

This leaves us with the possibilities $d=0$ or $d=1$. We next rely on the following

6.4. Lemma ([10]). Let $d \in \mathbb{N}$ and let $v: \mathbb{D} \rightarrow \mathbb{C}$ be a critical point of $E_{\infty}$ in $\mathscr{E}_{d}$.

1. If $d=0$, then $v$ is a constant of modulus 1 .

2. If $d \geq 1$, then $v$ is a Blaschke product; more specifically, $v$ is a product of d Moebius transforms.

The proof relies on the properties of the Hopf differential; see e.g. [25].

Using the above lemma combined with the fact that $d \in\{0,1\}$ and $v(0)=0$, we find that (possibly up to a subsequence)

(6.11) $v_{j} \rightarrow v=\alpha$ Id for some $\alpha \in \mathbb{S}^{1}$.

This, combined with the fact that $m_{r, \varepsilon}>\pi$, contradicts the next result. This contradiction completes the proof of Theorem 6.1.

6.5. Lemma. We have

$$
\int_{\mathbb{D}}\left|\nabla v_{j}\right|^{2} \rightarrow \int_{\mathbb{D}}|\nabla v|^{2}=2 \pi
$$

and

(6.13) $\int_{\mathbb{D}} w_{j}\left(1-\left|v_{j}\right|^{2}\right)^{2} \rightarrow 0$

\footnotetext{
${ }^{12}$ With more work, this approach gives also the stronger conclusion that Palais-Smale sequences are strongly compact.
} 
Proof. It suffices to establish (6.12)-(6.13) along a subsequence. We may assume that $\alpha=1$.

By Theorem 6.25 , there exists some $\lambda>0$ such that

(6.14) $\left|v_{j}(z)\right| \geq \lambda \quad \forall z \in \mathbb{D}$ such that $|z| \geq 1-\lambda$.

By standard elliptic estimates,

(6.15) $v_{j} \rightarrow v$ in $W_{l o c}^{2, p}(\mathbb{D}), \forall p<\infty$.

By (6.9) and (6.15), we find that (6.12) and (6.13) hold if we replace $\mathbb{D}$ by $\mathbb{D}_{r}$ for each $r<1$. Therefore, it suffices to prove that

(6.16) $\lim _{r \rightarrow 1} \limsup _{j \rightarrow \infty} \int_{\mathbb{D} \backslash \overline{\mathbb{D}}_{r}}\left(\left|\nabla v_{j}\right|^{2}+w_{j}\left(1-\left|v_{j}\right|^{2}\right)^{2}\right)=0$.

By (6.15), $v_{j} \rightarrow v$ uniformly on compacts of $\mathbb{D}$. Combining this fact with (6.14), we find that for large $j$ we have

$$
\operatorname{deg}\left(\frac{v_{j}}{\left|v_{j}\right|}, C(0, r)\right)=\operatorname{deg}\left(\frac{v}{|v|}, C(0, r)\right)=1, \quad \forall r \in[1-\lambda, 1] .
$$

Therefore, we may write, in $\omega=\mathbb{D} \backslash \overline{\mathbb{D}}_{1-\lambda}, v_{j}=\rho_{j} e^{\imath\left(\theta+\varphi_{j}\right)}$, with $\lambda \leq \rho_{j} \leq 1$. Possibly after extracting suitable multiples of $2 \pi$, we have $\varphi_{j} \rightarrow 0$ and $\rho_{j} \rightarrow \rho:=|v|$ in $H^{1}(\omega)$. On the other hand, by (6.15), we have $\varphi_{j} \rightarrow 0$ and $\rho_{j} \rightarrow \rho$ in $C_{l o c}^{1}(\omega)$.

We next translate the properties of $v_{j}$ in terms of $\rho_{j}$ and $\varphi_{j}$. We have

$$
\left\{\begin{array}{ll}
\operatorname{div}\left(\rho_{j}^{2} \nabla\left(\theta+\varphi_{j}\right)\right)=0 & \text { in } \omega \\
-\Delta \rho_{j}=w_{j} \rho_{j}\left(1-\rho_{j}\right)^{2}-\rho_{j}\left|\nabla\left(\theta+\varphi_{j}\right)\right|^{2} & \text { in } \omega \\
v_{j} \wedge \nabla v_{j}=\rho_{j}^{2} \nabla\left(\theta+\varphi_{j}\right) & \text { in } \omega \\
\left|\int_{\mathbb{D}}\left(v_{j} \wedge \nabla v_{j}\right) \cdot \nabla \zeta\right| \leq o(1)\|\nabla \zeta\|_{L^{2}}, & \forall \zeta \in H^{1}(\mathbb{D})
\end{array} .\right.
$$

Let $0<\delta<\lambda$. Since $\varphi_{j} \rightarrow 0$ in $C^{1}\left(C_{1-\delta}\right)$, we find that that the function $\varphi_{j}$, defined in $\mathbb{D} \backslash \overline{\mathbb{D}}_{1-\delta}$, has an extension $\zeta_{j} \in H^{1}(\mathbb{D})$ such that $\left\|\nabla \zeta_{j}\right\|_{L^{2}\left(\mathbb{D}_{1-\delta}\right)} \rightarrow 0$. Using the fact that

$$
\rho_{j}^{2} \nabla \theta \rightarrow \rho^{2} \nabla \theta \text { and } \rho_{j}^{2} \nabla \varphi_{j} \rightarrow 0 \quad \text { in } L^{2}(\omega),
$$

we find that

$$
\begin{aligned}
0 & =\lim _{j \rightarrow \infty} \int_{\mathbb{D}}\left(v_{j} \wedge \nabla v_{j}\right) \cdot \nabla \zeta_{j}=\lim _{j \rightarrow \infty} \int_{\mathbb{D}_{\mathbb{D}} \overline{\mathbb{D}}_{1-\delta}}\left(v_{j} \wedge \nabla v_{j}\right) \cdot \nabla \zeta_{j} \\
& =\lim _{j \rightarrow \infty} \int_{\mathbb{D}_{\mathbb{D}} \overline{\mathbb{D}}_{1-\delta}}\left[\rho_{j}^{2} \nabla\left(\theta+\varphi_{j}\right)\right] \cdot \nabla \varphi_{j}=\lim _{j \rightarrow \infty} \int_{\mathbb{D}_{\mathbb{D}} \overline{\mathbb{D}}_{1-\delta}} \rho_{j}^{2}\left|\nabla \varphi_{j}\right|^{2},
\end{aligned}
$$

which implies easily that

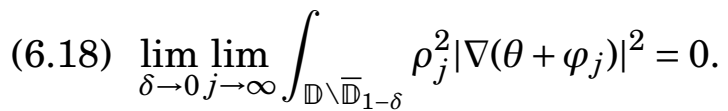

We next multiply by $\eta_{j}=1-\rho_{j}$ the equation satisfied by $\rho_{j}$ and find that

$$
\int_{\mathbb{D} \backslash \overline{\mathbb{D}}_{1-\delta}}\left(\left|\nabla \rho_{j}\right|^{2}+\frac{1+\rho_{j}}{\rho_{j}} w_{j}\left(1-\left|v_{j}\right|^{2}\right)^{2}\right)=\int_{\mathbb{D}_{\mathbb{D}_{1-\delta}}} \rho_{j} \eta_{j}\left|\nabla\left(\theta+\varphi_{j}\right)\right|^{2}+\int_{C_{1-\delta}} \eta_{j} \frac{\partial \rho_{j}}{\partial v},
$$

$v$ being the normal exterior to $\mathbb{D}_{1-\delta}$.

We next note that

(6.20) $\lim _{\delta \rightarrow 0} \lim _{j \rightarrow \infty} \int_{C_{1-\delta}} \eta_{j} \frac{\partial \rho_{j}}{\partial v}=\lim _{\delta \rightarrow 0} \int_{C_{1-\delta}}(1-\rho) \frac{\partial \rho}{\partial v}=0$.

By combining (6.19) with (6.18), (6.20) and with (6.14), we find that

(6.21) $\lim _{\delta \rightarrow 0} \limsup _{j \rightarrow \infty} \int_{\mathbb{D} \backslash \overline{\mathbb{D}}_{1-\delta}}\left(\left|\nabla \rho_{j}\right|^{2}+w_{j}\left(1-\left|v_{j}\right|^{2}\right)^{2}\right)=0$. 
Noting that $\left|\nabla v_{j}\right|^{2}=\left|\nabla \rho_{j}\right|^{2}+\rho_{j}\left|\nabla\left(\theta+\varphi_{j}\right)\right|^{2}$ in $\omega$, we obtain Lemma 6.5 by combining (6.18) with (6.21).

So far, this concerns the case where $\varepsilon$ is large. In Section 8, we discuss the case where $\varepsilon$ is small.

\section{ASYMPTOTICS AND UNIQUENESS}

We start with an asymptotic result when $\varepsilon \rightarrow 0$ in thin domains. This is a generalization of [8, Sections 8, 9, 10].

\subsection{Theorem. Assume that $\Omega$ is thin.}

1. Then (possibly up to subsequences) $u_{\varepsilon} \rightarrow u$ in $C^{1}(\bar{\Omega})$, where $u \in H^{1}\left(\Omega ; \mathbb{S}^{1}\right)$ is a minimizer of $\mathbf{m}_{\mathbf{d}}$.

2. For small $\varepsilon, u_{\varepsilon}$ is unique modulo $\mathbb{S}^{1}$ : if $v_{\varepsilon}$ is another minimizer of $E_{\varepsilon}$, then $v_{\varepsilon}=\alpha u_{\varepsilon}$ for some $\alpha \in \mathbb{S}^{1}$. And conversely.

The same holds in critical domains such that $\mathbf{m}_{\mathbf{d}}=2 \pi$.

Sketch of proof. Step 1. Identification of the limit in $H^{1}(\Omega)$.

Assume first that $\Omega$ is thin. The proof of Theorem 4.12, and more specifically (4.22), implies that (possibly up to subsequences) $u_{\varepsilon} \rightarrow u$, where $u \in H^{1}\left(\Omega ; \mathbb{S}^{1}\right) \cap \mathscr{E}_{\mathbf{e}}$ and the balanced collection $\mathbf{e}$ satisfies

$$
\mathbf{m}_{\mathbf{e}}+\pi|\mathbf{d}-\mathbf{e}| \leq \frac{1}{2} \int_{\Omega}|\nabla u|^{2} \leq \liminf _{\varepsilon \rightarrow \infty} \frac{1}{2} \int_{\Omega}\left|\nabla u_{\varepsilon}\right|^{2} \leq \mathbf{m}_{\mathbf{d}} .
$$

Since $\Omega$ is thin, we find that $\mathbf{e}=\mathbf{d}$, and thus $u_{\varepsilon} \rightarrow u$ in $H^{1}$.

More delicate is the case where $\Omega$ is critical and $\mathbf{m}_{\mathbf{d}}=2 \pi$. The starting point is the following

7.2. Lemma. Let $\mathbf{d}$ be balanced and let $u$ be a minimizer of $\mathbf{m}_{\mathbf{d}}$. Let $\mathbf{m}_{\mathbf{d}, \varepsilon}^{*}$ be as in (5.2). Then

$$
\mathbf{m}_{\mathbf{d}, \varepsilon}^{*}=\mathbf{m}_{\mathbf{d}}-\frac{\varepsilon^{2}}{4} \int_{\Omega}|\nabla u|^{4}+o\left(\varepsilon^{2}\right) \quad \text { as } \varepsilon \rightarrow 0 .
$$

Proof of Lemma 7.2. By the proof of Proposition 5.1 and by Lemma 5.2, we may write, for small $\varepsilon$, $u_{\varepsilon}=\rho_{\varepsilon} u e^{l \varphi_{\varepsilon}}$, and then we have

$$
E_{\varepsilon}\left(u_{\varepsilon}\right)=\int_{\Omega}\left(\frac{1}{2}\left|\nabla \rho_{\varepsilon}\right|^{2}+\frac{1}{2} \rho_{\varepsilon}^{2}\left|u \wedge \nabla u+\nabla \varphi_{\varepsilon}\right|^{2}+\frac{1}{4 \varepsilon^{2}}\left(1-\rho_{\varepsilon}^{2}\right)^{2}\right)
$$

We will estimate each term in (7.3).

By Proposition 2.2, the smooth maps $\rho_{\varepsilon}$ and $\varphi_{\varepsilon}$ solve

$$
\left\{\begin{array}{ll}
\operatorname{div}\left(\rho_{\varepsilon}^{2}\left(u \wedge \nabla u+\nabla \varphi_{\varepsilon}\right)\right)=0 & \text { in } \Omega \\
-\Delta \rho_{\varepsilon}=\frac{1}{\varepsilon^{2}} \rho_{\varepsilon}\left(1-\rho_{\varepsilon}\right)^{2}-\rho_{\varepsilon}\left|u \wedge \nabla u+\nabla \varphi_{\varepsilon}\right|^{2} & \text { in } \Omega \\
\frac{\partial \varphi_{\varepsilon}}{\partial v}=0 & \text { on } \partial \Omega \\
\rho_{\varepsilon}=1 & \text { on } \partial \Omega
\end{array} .\right.
$$

The analysis of the system (7.4) developed in [8, Section 8] yields the convergences $\rho_{\varepsilon} \rightarrow 1, \varphi_{\varepsilon} \rightarrow 0$ in $C^{1, \beta}(\bar{\Omega}), 0<\beta<1$.

We claim that

$$
\int_{\Omega}\left|\nabla \rho_{\varepsilon}\right|^{2}+\frac{1}{\varepsilon^{2}} \int_{\Omega}\left(1-\rho_{\varepsilon}^{2}\right)^{2}=O\left(\varepsilon^{2}\right)
$$

Indeed, this is obtained by multiplying by $\rho_{\varepsilon}-1$ the equation of $\rho_{\varepsilon}$. Using the fact that, by the maximum principle, we have $\rho_{\varepsilon} \leq 1$, we find that

$$
\begin{aligned}
\int_{\Omega}\left(\left|\nabla \rho_{\varepsilon}\right|^{2}+\frac{1}{\varepsilon^{2}} \frac{\rho_{\varepsilon}}{1+\rho_{\varepsilon}}\left(1-\rho_{\varepsilon}^{2}\right)^{2}\right) & =\int_{\Omega} \rho_{\varepsilon}\left(1-\rho_{\varepsilon}\right)\left|u \wedge \nabla u+\nabla \varphi_{\varepsilon}\right|^{2} \\
& \leq \frac{1}{2 \varepsilon^{2}} \int_{\Omega}\left(1-\rho_{\varepsilon}\right)^{2}+C \varepsilon^{2},
\end{aligned}
$$

and this leads easily to the desired conclusion. 
We next rely on the interior estimates of Bethuel, Brezis and Hélein [13, Theorem 2].

7.3. Theorem ([13]). Assume that $u_{\varepsilon}$ minimizes $E_{\varepsilon}$ with respect to its own Dirichlet boundary condition, that $E_{\varepsilon}\left(u_{\varepsilon}\right) \leq C$ and that $u_{\varepsilon} \rightarrow u$ in $H^{1}(\Omega)$. Then

(7.7) $\frac{1-\left|u_{\varepsilon}\right|^{2}}{\varepsilon^{2}} \rightarrow|\nabla u|^{2}=|u \wedge \nabla u|^{2} \quad$ in $C^{\infty}(\Omega)$.

Let $\delta>0$ be small and fixed. By (7.7), we find that

$$
\int_{\Omega_{\delta}}\left(\frac{1}{2}\left|\nabla \rho_{\varepsilon}\right|^{2}+\frac{1}{4 \varepsilon^{2}}\left(1-\left|u_{\varepsilon}\right|^{2}\right)^{2}\right)=\frac{\varepsilon^{2}}{4} \int_{\Omega_{\delta}}|\nabla u|^{4}+o\left(\varepsilon^{2}\right) .
$$

On the other hand, if we repeat in $\Omega \backslash \Omega_{\delta}$ the argument leading to (7.5) and use (7.7), we find that

$$
\begin{aligned}
\int_{\Omega_{\delta}}\left(\left|\nabla \rho_{\varepsilon}\right|^{2}+\frac{1}{\varepsilon^{2}} \frac{\rho_{\varepsilon}}{1+\rho_{\varepsilon}}\left(1-\rho_{\varepsilon}^{2}\right)^{2}\right) & =\int_{\Omega_{\delta}} \rho_{\varepsilon}\left(1-\rho_{\varepsilon}\right)\left|u \wedge \nabla u+\nabla \varphi_{\varepsilon}\right|^{2}-\int_{\partial \Omega_{\delta}}\left(1-\rho_{\varepsilon}\right) \frac{\partial \rho_{\varepsilon}}{\partial v} \\
& \leq \frac{1}{2 \varepsilon^{2}} \int_{\Omega_{\delta}}\left(1-\rho_{\varepsilon}\right)^{2}+C\left(\delta \varepsilon^{2}+\varepsilon^{4}\right),
\end{aligned}
$$

and thus

$$
\int_{\Omega \backslash \Omega_{\delta}}\left|\nabla \rho_{\varepsilon}\right|^{2}+\frac{1}{\varepsilon^{2}} \int_{\Omega \backslash \Omega_{\delta}}\left(1-\rho_{\varepsilon}^{2}\right)^{2} \leq C \delta \varepsilon^{2}+o\left(\varepsilon^{2}\right) .
$$

By combining (7.8) with (7.10), we find that

$$
\int_{\Omega}\left(\frac{1}{2}\left|\nabla \rho_{\varepsilon}\right|^{2}+\frac{1}{4 \varepsilon^{2}}\left(1-\rho_{\varepsilon}^{2}\right)^{2}\right)=\frac{\varepsilon^{2}}{4} \int_{\Omega}|\nabla u|^{4}+o\left(\varepsilon^{2}\right) .
$$

In view of (7.3), it remains to prove that

$$
\frac{1}{2} \int_{\Omega} \rho_{\varepsilon}^{2}\left|u \wedge \nabla u+\nabla \varphi_{\varepsilon}\right|^{2}=\mathbf{m}_{\mathbf{d}}-\frac{\varepsilon^{2}}{2} \int_{\Omega}|\nabla u|^{4}+o\left(\varepsilon^{2}\right)=\frac{1}{2} \int_{\Omega}|\nabla u|^{2}-\frac{\varepsilon^{2}}{2} \int_{\Omega}|\nabla u|^{4}+o\left(\varepsilon^{2}\right) .
$$

By the argument leading to (7.11), we have

$$
\frac{1}{2} \int_{\Omega} \rho_{\varepsilon}^{2}|\nabla u|^{2}=\frac{1}{2} \int_{\Omega}|\nabla u|^{2}-\frac{\varepsilon^{2}}{2} \int_{\Omega}|\nabla u|^{4}+o\left(\varepsilon^{2}\right),
$$

and also

(7.12) $\int_{\Omega}\left(1-\rho_{\varepsilon}^{2}\right)|\nabla u|^{2}=o\left(\varepsilon^{2}\right) \quad$ and $\quad \int_{\Omega}\left(1-\rho_{\varepsilon}^{2}\right)\left|u \wedge \nabla u+\nabla \varphi_{\varepsilon}\right|^{2}=o\left(\varepsilon^{2}\right)$.

Therefore, it suffices to prove that

$$
\int_{\Omega}\left(\left|u \wedge \nabla u+\nabla \varphi_{\varepsilon}\right|^{2}-|\nabla u|^{2}\right)=o\left(\varepsilon^{2}\right)
$$

Estimate (7.13) is obtained by combining (7.12) with the fact that

$$
2 \mathbf{m}_{\mathbf{d}}=\int_{\Omega}|\nabla u|^{2} \leq \int_{\Omega}\left|\nabla\left(u e^{i \varphi_{\varepsilon}}\right)\right|^{2}=\int_{\Omega}\left|u \wedge \nabla u+\nabla \varphi_{\varepsilon}\right|^{2}
$$

and with the inequality

$$
\int_{\Omega} \rho_{\varepsilon}^{2}\left|u \wedge \nabla u+\nabla \varphi_{\varepsilon}\right|^{2} \leq \int_{\Omega} \rho_{\varepsilon}^{2}|\nabla u|^{2},
$$

which is a consequence of (7.4).

Step 1 continued. Assume that $\Omega$ is critical and that $\mathbf{m}_{\mathbf{d}}=2 \pi$. By (7.1), we find that either $\mathbf{e}=\mathbf{d}$ (and then we continue as in the thin case), or $\mathbf{e}=0$. In the latter case, $|\mathbf{d}|=2$ and $u$ is a constant of modulus 1 . Therefore, we may invoke (4.37) and find that

(7.14) $\mathbf{m}_{\mathbf{d}, \varepsilon} \geq 2 \pi-C_{4} e^{-C_{5} / \varepsilon}$.

Since $\mathbf{m}_{\mathbf{d}, \varepsilon} \leq \mathbf{m}_{\mathbf{d}, \varepsilon}^{*},(7.14)$ contradicts Lemma 7.2. 
Step 2. Stronger convergence.

By Lemma 5.2, we have $\left|u_{\varepsilon}\right| \rightarrow 1$ uniformly in $\Omega$ as $\varepsilon \rightarrow 0$. As in the proof of Lemma 7.2, we may transform the $H^{1}$ convergences into the stronger convergences $\varphi_{\varepsilon} \rightarrow 0, \rho_{\varepsilon} \rightarrow 1$ in $C^{1, \beta}(\bar{\Omega}), 0<\beta<1$ [8, Section 8], and thus $u_{\varepsilon} \rightarrow u$ in $C^{1, \beta}(\bar{\Omega})$.

Step 3. Uniqueness.

This follows essentially from the method developed in [28]. Write, for small $\varepsilon, v_{\varepsilon}=u_{\varepsilon} \eta_{\varepsilon} e^{\imath \psi_{\varepsilon}}$. The starting point is the identity [28, Substitution lemma]

$$
\begin{aligned}
E_{\varepsilon}\left(v_{\varepsilon}\right)= & E_{\varepsilon}\left(u_{\varepsilon}\right)+\frac{1}{2} \int_{\Omega}\left|u_{\varepsilon}\right|^{2}\left|\nabla \eta_{\varepsilon}\right|^{2}+\frac{1}{2} \int_{\Omega}\left|v_{\varepsilon}\right|^{2}\left|\nabla \psi_{\varepsilon}\right|^{2} \\
& +\underbrace{\int_{\Omega}\left(\eta_{\varepsilon}^{2}-1\right)\left(u_{\varepsilon} \wedge \nabla u_{\varepsilon}\right) \cdot \nabla \psi_{\varepsilon}}_{I_{\varepsilon}}+\frac{1}{4 \varepsilon^{2}} \int_{\Omega}\left|u_{\varepsilon}\right|^{4}\left(1-\eta_{\varepsilon}^{2}\right)^{2} .
\end{aligned}
$$

By Cauchy-Schwarz and the fact that $u_{\varepsilon}, v_{\varepsilon} \rightarrow u$ in $C^{1}(\bar{\Omega})$, we have

$$
\left|I_{\varepsilon}\right| \leq \frac{1}{8 \varepsilon^{2}} \int_{\Omega}\left|u_{\varepsilon}\right|^{4}\left(1-\eta_{\varepsilon}^{2}\right)^{2}+C \varepsilon^{2} \int_{\Omega}\left|v_{\varepsilon}\right|^{2}\left|\nabla \psi_{\varepsilon}\right|^{2},
$$

and thus, for small $\varepsilon, E_{\varepsilon}\left(v_{\varepsilon}\right)>E_{\varepsilon}\left(u_{\varepsilon}\right)$ unless $\eta_{\varepsilon}=1$ and $\psi_{\varepsilon}$ is a constant, which amounts to $v_{\varepsilon}=\alpha u_{\varepsilon}$ with $\alpha \in \mathbb{S}^{1}$.

In a different but related direction, we mention without proof the following result, stated for convenience in $\Omega=\mathbb{D}$ for the energy $E_{\varepsilon}(u)=\frac{1}{2} \int_{\mathbb{D}}|\nabla u|^{2}+\frac{1}{4 \varepsilon^{2}} \int_{\mathbb{D}} w\left(1-|u|^{2}\right)^{2}$.

7.4. Theorem ([10]). Let $u_{\varepsilon}$ be a minimax critical point as in the proof of Theorem 6.1. Then, as $\varepsilon \rightarrow \infty, u_{\varepsilon}$ converges (possibly up to a subsequence) strongly in $H^{1}(\mathbb{D})$ to a solution of

$$
\max \left\{\int_{\mathbb{D}} w\left(1-\left|M_{\alpha, a}\right|^{2}\right)^{2} ; \alpha \in \mathbb{S}^{1}, a \in \mathbb{D}\right\}
$$

We next present a non asymptotic result from [23], which is a considerable improvement of a result of Golovaty and Berlyand [24] concerning uniqueness in circular annuli.

For $s>0$, we denote by $\mathscr{A}_{s}$ the area of $\{z \in \mathbb{D} ; \operatorname{Re} z>s\}$. It is easy to see that there is exactly one $\sigma \in(0,1)$ such that $\mathscr{A}_{\sigma}=\frac{\sigma^{6}}{32}$. For this $\sigma$, we set $\delta=2 \mathscr{A}_{\sigma} \cdot{ }^{13}$

7.5. Definition. A balanced collection $\mathbf{d}$ is $\operatorname{slim}$ if $\mathbf{m}_{\mathbf{d}} \leq \delta$.

Returning to the examples considered in Section 4, we see that a doubly connected domain with $\mathbf{d}=(d, d)$ is slim provided $R<R(d)$ with $R(d)$ sufficiently close to 1 , and that $\Omega_{t}$ in Fig. 2 is slim provided $t>t(\mathbf{d})$. On the other hand, it is easy to see that slim implies thin.

By (4.23), if $\Omega$ is slim then

(7.17) $\mathbf{m}_{\mathbf{d}, \varepsilon}<\delta \quad \forall \varepsilon>0$.

This inequality will be crucial in what follows. Before going further, let us mention that (7.17) implies that $\mathbf{d}$ is balanced. Indeed, this follows from the fact that, if $\mathbf{d}$ is unbalanced and $u \in \mathscr{E}_{\mathbf{d}}$, then

$$
\mathbf{m}_{\mathbf{d}, \varepsilon} \geq \frac{1}{2} \int_{\Omega}|\nabla u|^{2} \geq\left|\int_{\Omega} \operatorname{Jac} u\right|=\pi\left|d_{0}-\sum_{j \geq 1} d_{j}\right| \geq \pi>\delta .
$$

7.6. Theorem ([23]). Let $\Omega$ be slim. Then (modulo $\left.\mathbb{S}^{1}\right) E_{\varepsilon}$ has exactly a minimizer in $\mathscr{E}_{\mathbf{d}}$.

Note that, since $\mathbf{m}_{\mathbf{d}} \leq 2 \pi$, existence of a minimizer follows from Proposition 4.13 , so that the issue is to prove uniqueness. The proof of the uniqueness relies on the following special case of [23, Theorem 3.6].

\footnotetext{
${ }^{13}$ The approximate value of $\delta$ is $\delta=0.04518303544 \ldots$
} 
7.7. Theorem. Let $u_{\varepsilon}$ minimize $E_{\varepsilon}$ in $\mathscr{E}_{\mathbf{d}}$ and let $p \in(0,1)$. If $\frac{1}{2} \int_{\Omega}|\nabla u|^{2} \leq 2 \mathscr{A}_{p}$, then $|u| \geq p$ in $\Omega$.

The proof of Theorem 7.7 is delicate and will not be presented here. However, in order to give a flavor of the proof, we present a much simpler related result.

7.8. Theorem ([23]). Let $\Omega$ be simply connected and let $p \in(0,1)$. Let $u$ minimize $E_{\varepsilon}$ subject to $\operatorname{tr} u=g \in C^{1}\left(\partial \Omega ; \mathbb{S}^{1}\right)$. If $\frac{1}{2} \int_{\Omega}|\nabla u|^{2} \leq 2 \mathscr{A}_{p}$, then $|u| \geq p$ in $\Omega$.

Note the following interesting

7.9. Corollary. Let $\Omega$ be simply connected. Let $u$ minimize $E_{\varepsilon}$ subject to $\operatorname{tr} u=g \in C^{1}\left(\partial \Omega ; \mathbb{S}^{1}\right)$. If $\int_{\Omega}|\nabla u|^{2}<\pi$, then u does not vanish in $\Omega$.

Proof of Theorem 7.8. Step 1. The level sets of $|u|$ are "long".

Let $m=\min _{\Omega}|u|$. Let $z_{0}$ be such that $\left|u\left(z_{0}\right)\right|=m$. For a generic $t \in(m, 1)$, the level set $[|u|=t]$ is smooth, and contains a closed curve $\gamma_{t}$ enclosing $z_{0}$. Consider the circle arc $\mathscr{C}_{t}=u\left(\gamma_{t}\right) \subset C(0, t)$. Let $\ell_{t, s}$ be the length of $C(0, t) \cap\{\operatorname{Re} z>s\}$. We claim that the length of $\mathscr{C}_{t}$ is at least $\ell_{t, m}$. Indeed, argue by contradiction. Up to a rotation, we may assume that $\mathscr{C}_{t}$ is contained in $C(0, r) \cap\{\operatorname{Re} z>s\}$ for some $s>m$. As we will see, this implies

(7.18) $|u| \geq s$ at the interior of $\gamma_{t}$;

this conclusion contradicts the fact that $z_{0}$ is at the interior of $\gamma_{t}$ and $\left|u\left(z_{0}\right)\right|=m<s$.

It remains to prove (7.18). Consider the set $S=\{z \in \overline{\mathbb{D}} ; \operatorname{Re} z \geq s\}$. Let $\omega$ denote the interior of $\gamma_{t}$ and set $h=u_{\mid \partial \omega}$, whose image lies inside $S$. Our aim is to prove that $u(\omega) \subset S$.

The map $v=|\operatorname{Re} u|+\imath \operatorname{Im} u$ equals $h$ on $\partial \omega$ and has the same energy as $u$. Thus $v$ minimizes $E_{\varepsilon}$ in $\omega$ with boundary datum $h$. Since $\omega$ is connected, if we prove that $v(\omega) \subset S$, then we also have $u(\omega) \subset S$. We thus reduced the problem to the case where Re $u \geq 0$.

Let $\Pi$ be the orthogonal projection on $S$. The following is straightforward.

(7.19) $|z| \leq|\Pi(z)| \leq 1$ for $z \in \overline{\mathbb{D}} \cap\{\operatorname{Re} z \geq 0\}$.

Set $w=\Pi \circ u$, which equals $h$ on $\partial \omega$. Since $\Pi$ is a contraction, we have $|\nabla w| \leq|\nabla u|$. This fact combined with (7.19), and with the fact that $u$ minimizes $E_{\varepsilon}$ in $\omega$ with boundary datum $h$ implies that $w$ is a minimizer of $E_{\varepsilon}$ in $\omega$ with boundary datum $h$. Actually, a bit more can be said. Indeed, by combining the minimality of $u$ with the fact that $|\nabla w| \leq|\nabla u|$ and with the inequality $\left(1-|w|^{2}\right)^{2} \leq\left(1-|u|^{2}\right)^{2}$, we come up with the equalities $|\nabla w|=|\nabla u|$ and $|w|=|u|$.

Consider now the open set

$$
V=\{x \in \omega ; u(x) \notin S\}=\{x \in \omega ; \operatorname{Re} u<s\} .
$$

Our aim is to prove that $V$ is empty. Let $\Pi=\Pi_{1}+\imath \Pi_{2}$ and set

$$
F=\{z \in \overline{\mathbb{D}} \backslash S ; \operatorname{Re} z \geq 0\} .
$$

If $z \in F$, then $\Pi_{1}(z)=s$ and $\Pi_{2}(z)$ depends only on $\operatorname{Im} z$. On the other hand, we have

$$
\left|\Pi_{2}(z)-\Pi_{2}(\xi)\right| \leq|\operatorname{Im} z-\operatorname{Im} \xi|, \quad \forall z, \xi \in F .
$$

By the above, we have $\nabla(\operatorname{Re} w)=0$ and $|\nabla(\operatorname{Im} w)| \leq|\nabla(\operatorname{Im} u)|$ in $V$. Since we also have $|\nabla w|=|\nabla u|$, we find that $\nabla(\operatorname{Re} u)=0$ in $V$. Consequently, $\operatorname{Re} u$ is locally constant in $V$.

Assuming, by contradiction, that $V$ is not empty, we claim that Re $u=s$ on $\partial V$. Indeed, let $V_{0}$ be a connected component of $V$. If $x \in \partial V_{0}$, then either $u(x) \in S$, or $x \in \partial \omega$. In the first case, the definition of $V$ implies $\operatorname{Re} u(x)=s$; in the latter, we obtain the same conclusion via the fact that $h \in C^{0}(\partial \omega ; \mathbb{C})$. Since $\operatorname{Re} u$ is locally constant in $V$, we find that $\operatorname{Re} u=s$ in $V$. This contradiction completes the proof of (7.18).

Step 2. The Dirichlet energy of $u$ is "large".

Consider again a generic $t \in(m, 1)$. Write, locally in the set $U=\{x \in \Omega ; u(x) \neq 0\}, u$ in the form 
$u=\rho e^{\imath \varphi}$, with $\rho=|u|$ and $\varphi$ real-valued and $C^{1}$. On the one hand, we have

(7.20) $|\nabla u|^{2}=|\nabla \rho|^{2}+\rho^{2}|\nabla \varphi|^{2} \geq 2 \rho|\nabla \varphi||\nabla \rho| \quad$ in $U$.

By (7.20) and the coarea formula, we find that

$$
\frac{1}{2} \int_{U}|\nabla u|^{2} \geq \int_{m}^{1}\left(\int_{[\rho=t]} \rho|\nabla \varphi|\right) d t=\int_{m}^{1} t\left(\int_{[\rho=t]}|\nabla \varphi|\right) d t
$$

On the other hand, we claim that for a generic $t$ we have

$$
\int_{[\rho=t]}|\nabla \varphi|=\int_{[\rho=t]}\left|\left(\frac{u}{|u|}\right) \wedge \nabla\left(\frac{u}{|u|}\right)\right| \geq \int_{[\rho=t]}\left|\left(\frac{u}{|u|}\right) \wedge \frac{\partial}{\partial \tau}\left(\frac{u}{|u|}\right)\right| \geq 2 \frac{\ell_{t, m}}{t} .
$$

Only the last inequality in (7.22) requires an explanation. Assume first that $u\left(\gamma_{t}\right)$ is strictly contained in $C(0, t)$. Let $A, B \in \gamma_{t}$ be such that the endpoints of $u\left(\gamma_{t}\right)$ are $u(A)$ and $u(B)$. Let $\mathscr{D}_{j}$, $j=1,2$, be the two arcs of $\gamma_{t}$ with endpoints $A$ and $B$. Write, on each $\mathscr{D}_{j}, u=t e^{l \varphi_{j}}$; this time, $\varphi_{j}$ is not only locally, but globally defined. Since the quantity $t\left|\varphi_{j}(A)-\varphi_{j}(B)\right|$ equals the length of $\left(u\left(\gamma_{t}\right)\right)$, we find, via Step 1, that

$$
\int_{[\rho=t]}\left|\left(\frac{u}{|u|}\right) \wedge \frac{\partial}{\partial \tau}\left(\frac{u}{|u|}\right)\right|=\sum_{j=1}^{2} \int_{\mathscr{D}_{j}}\left|\frac{\partial \varphi_{j}}{\partial \tau}\right| \geq\left|\varphi_{1}(A)-\varphi_{1}(B)\right|+\left|\varphi_{2}(A)-\varphi_{2}(B)\right| \geq 2 \frac{\ell_{t, m}}{t},
$$

i.e., (7.22) holds.

Assume next that $u\left(\gamma_{t}\right)=C(0, t)$. Then the variation of $\frac{u}{|u|}$ on $\gamma_{t}$ is at least $2 \pi$, and thus

$$
\int_{[\rho=t]}\left|\left(\frac{u}{|u|}\right) \wedge \frac{\partial}{\partial \tau}\left(\frac{u}{|u|}\right)\right| \geq\left|\int_{[\rho=t]} \frac{\partial}{\partial \tau}\left(\frac{u}{|u|}\right)\right| \geq 2 \pi,
$$

so that again (7.22) holds.

By combining (7.21) with (7.22) and applying the coarea formula, we find that

$$
\frac{1}{2} \int_{\Omega}|\nabla u|^{2} \geq 2 \int_{m}^{1} \ell_{t, m} d t=2 \mathscr{A}_{m}
$$

Since by assumption $\frac{1}{2} \int_{\Omega}|\nabla u|^{2} \leq 2 \mathscr{A}_{p}$, we find that $\mathscr{A}_{m} \leq \mathscr{A}_{p}$, and thus $m \geq p$. Consequently, $|u| \geq p$ in $\Omega$.

Proof of Theorem 7.6. Let $u_{\varepsilon}, v_{\varepsilon}$ be two minimizers of $E_{\varepsilon}$ in $\mathscr{E}_{\mathbf{d}}$. By Theorem 7.6, we have $\left|u_{\varepsilon}\right| \geq \sigma$ and $\left|v_{\varepsilon}\right| \geq \sigma$. We write $v_{\varepsilon}=u_{\varepsilon} \eta_{\varepsilon} e^{\imath \psi_{\varepsilon}}$. By (2.4), the smooth vector field $u_{\varepsilon} \wedge \nabla u_{\varepsilon}$ satisfies

$$
\left\{\begin{array}{ll}
\operatorname{div}\left(u_{\varepsilon} \wedge \nabla u_{\varepsilon}\right)=0 & \text { in } \Omega \\
\left(u_{\varepsilon} \wedge \nabla u_{\varepsilon}\right) \cdot v=0 & \text { on } \partial \Omega
\end{array} .\right.
$$

Therefore, we may find a global function $H_{\varepsilon}$ such that $\partial_{x} H_{\varepsilon}=u_{\varepsilon} \wedge \partial_{y} u_{\varepsilon}$ and $\partial_{y} H_{\varepsilon}=-u_{\varepsilon} \wedge \partial_{x} u_{\varepsilon}$. Inserting $H_{\varepsilon}$ into the identity (7.15), we find that

$$
\begin{aligned}
E_{\varepsilon}\left(v_{\varepsilon}\right)= & E_{\varepsilon}\left(u_{\varepsilon}\right)+\frac{1}{2} \int_{\Omega}\left|u_{\varepsilon}\right|^{2}\left|\nabla \eta_{\varepsilon}\right|^{2}+\frac{1}{2} \int_{\Omega}\left|v_{\varepsilon}\right|^{2}\left|\nabla \psi_{\varepsilon}\right|^{2} \\
& -\int_{\Omega}\left(1-\eta_{\varepsilon}^{2}\right) \operatorname{Jac}\left(H_{\varepsilon}, \psi_{\varepsilon}\right)+\frac{1}{4 \varepsilon^{2}} \int_{\Omega}\left|u_{\varepsilon}\right|^{4}\left(1-\eta_{\varepsilon}^{2}\right)^{2} .
\end{aligned}
$$

Using the fact that $\sigma \leq\left|u_{\varepsilon}\right|,\left|v_{\varepsilon}\right| \leq 1$, we obtain

$$
E_{\varepsilon}\left(v_{\varepsilon}\right) \geq E_{\varepsilon}\left(u_{\varepsilon}\right)+\frac{\sigma^{2}}{2} \int_{\Omega}\left(\left|\nabla \eta_{\varepsilon}\right|^{2}+\left|\nabla \psi_{\varepsilon}\right|^{2}\right)-\left|\int_{\Omega}\left(1-\eta_{\varepsilon}^{2}\right) \operatorname{Jac}\left(H_{\varepsilon}, \psi_{\varepsilon}\right)\right|+\frac{1}{4 \varepsilon^{2}} \int_{\Omega}\left|u_{\varepsilon}\right|^{4}\left(1-\eta_{\varepsilon}^{2}\right)^{2} .
$$

We next invoke the celebrated Wente estimates in the quantitative form of Bethuel and Ghidaglia.

7.10. Theorem ([15]). Let $f \in H_{0}^{1}(\Omega)$ and $g, h \in H^{1}(\Omega)$. Then

$$
\left|\int_{\Omega} f \operatorname{Jac}(g, h)\right| \leq \sqrt{2}\|\nabla f\|_{L^{2}}\|\nabla g\|_{L^{2}}\|\nabla h\|_{L^{2}} \text {. }
$$


By combining (7.25) with (7.24) and with the fact that

$$
\left\|\nabla H_{\varepsilon}\right\|_{L^{2}}=\left\|u_{\varepsilon} \wedge \nabla u_{\varepsilon}\right\|_{L^{2}} \leq\left\|\nabla u_{\varepsilon}\right\|_{L^{2}} \leq \sqrt{2 \delta},
$$

we find that

$$
E_{\varepsilon}\left(v_{\varepsilon}\right) \geq E_{\varepsilon}\left(u_{\varepsilon}\right)+\frac{\sigma^{2}}{2} \int_{\Omega}\left(\left|\nabla \eta_{\varepsilon}\right|^{2}+\left|\nabla \psi_{\varepsilon}\right|^{2}\right)-\frac{4 \sqrt{\delta}}{\sigma}\left\|\nabla \eta_{\varepsilon}\right\|_{L^{2}}\left\|\nabla \psi_{\varepsilon}\right\|_{L^{2}}+\frac{1}{4 \varepsilon^{2}} \int_{\Omega}\left|u_{\varepsilon}\right|^{4}\left(1-\eta_{\varepsilon}^{2}\right)^{2} .
$$

Our choice of $\sigma$ implies that $E_{\varepsilon}\left(v_{\varepsilon}\right) \geq E_{\varepsilon}\left(u_{\varepsilon}\right)$, with equality if and only if $v_{\varepsilon}=\alpha u_{\varepsilon}$ for some $\alpha \in$ $\mathbb{S}^{1}$.

\section{PERSPECTIVES AND OPEN PROBLEMS}

As already mentioned in Section 4, we propose the following

Conjecture 1. Assume that $\Omega$ is thick. Then, for small $\varepsilon$, the infimum $\mathbf{m}_{\mathbf{d}, \varepsilon}$ is not attained.

We note that, for large $\varepsilon, \mathbf{m}_{\mathbf{d}, \varepsilon}$ may be attained. For example, if $\Omega$ is doubly connected and $\mathbf{d}=(1,1)$, then $\mathbf{m}_{\mathbf{d}, \varepsilon}$ is attained for large $\varepsilon$, though $\Omega$ is thick for large $R$ [8, Corollary 5.5].

Conjecture 1 is known to be true only for fat domains. An inspection of the proof of Theorem 4.16 reveals that an important step is concentration of energy near boundary. This suggests the following.

Conjecture 2. Let $u_{\varepsilon}$ minimize $E_{\varepsilon}$ in $\mathscr{E}_{\mathbf{d}}$. If $u_{\varepsilon} \rightarrow u$ as $\varepsilon \rightarrow 0$, with $u \in \mathscr{E}_{\mathbf{e}}$ and $\mathbf{e} \neq \mathbf{d}$, then

$$
\mathbf{m}_{\mathbf{d}, \varepsilon}=E_{\varepsilon}\left(u_{\varepsilon}\right) \geq \mathbf{m}_{\mathbf{e}, \varepsilon}+\pi|\mathbf{d}-\mathbf{e}|+o\left(\varepsilon^{2}\right) .
$$

A stronger conjecture, implying the two first ones, is the following.

Conjecture 3. Let $u_{\varepsilon}$ minimize $E_{\varepsilon}$ in $\mathscr{E}_{\mathbf{d}}$. If $u_{\varepsilon} \rightarrow u$ as $\varepsilon \rightarrow 0$, then $u \in \mathscr{E}_{\mathbf{d}}$.

We continue by presenting a consequence of Conjecture 2 on existence of minimizers.

Consider a critical domain. Let $\mathscr{B}^{*}$ be the non empty collection

$$
\mathscr{B}^{*}:=\left\{\mathbf{e} \in \mathscr{B} \backslash\{\mathbf{d}\} ; \mathbf{m}_{\mathbf{d}}=\mathbf{m}_{\mathbf{e}}+\pi|\mathbf{d}-\mathbf{e}|\right\} .
$$

8.1. Proposition. Assume that Conjecture 2 holds. Let $\Omega$ be a doubly connected critical domain. Then, for small $\varepsilon, \mathbf{m}_{\mathbf{d}, \varepsilon}$ is attained by some $u_{\varepsilon}$, and (possibly after a rotation) we have $u_{\varepsilon} \rightarrow u^{\mathbf{d}}$.

Proof. Consider, for fixed small $\varepsilon$, a minimizing sequence $\left(u_{n, \varepsilon}\right)$ weakly converging to some $u_{\varepsilon}$. As in the proof of Theorem 4.12, we may assume that $u_{\varepsilon} \in \mathscr{E}_{\mathbf{f}}$, with $\mathbf{f}$ independent of $\varepsilon$, and that $u_{\varepsilon} \rightarrow u$, where $|u|=1$ and $u \in \mathscr{E}_{\mathbf{e}}$. As in the proof of Theorem $4.12, u_{\varepsilon}$ minimizes $E_{\varepsilon}$ in $\mathscr{E}_{\mathbf{f}}$ and we have

$$
\mathbf{m}_{\mathbf{d}} \geq \mathbf{m}_{\mathbf{d}, \varepsilon}=\mathbf{m}_{\mathbf{f}, \varepsilon}+\pi|\mathbf{d}-\mathbf{f}| \geq \mathbf{m}_{\mathbf{e}}+\pi(|\mathbf{d}-\mathbf{f}|+|\mathbf{f}-\mathbf{e}|)+o(1) .
$$

If $\mathbf{e}=\mathbf{d}$, then $\mathbf{e}=\mathbf{f}=\mathbf{d}$, and we are done. Otherwise, noting that $\mathbf{e}$ is balanced, we find that $\mathbf{e} \in \mathscr{B}^{*}$ and

$$
|\mathbf{d}-\mathbf{f}|+|\mathbf{f}-\mathbf{e}|=|\mathbf{d}-\mathbf{e}| .
$$

We now take advantage of the fact that $\Omega$ is doubly connected. Let $\mathbf{d}=(d, d)$ with, say, $d>0$. Then $\mathbf{e}=(d-1, d-1) .{ }^{14}$ Since $\mathbf{d}$ is critical, Proposition 4.8 implies that $\mathbf{e}$ is thin. By Theorem 7.1 combined with the proof of Proposition 5.1 and with Lemma 7.2 we find that, for small $\varepsilon$, we have

$$
\mathbf{m}_{\mathbf{e}, \varepsilon}=\mathbf{m}_{\mathbf{e}, \varepsilon}^{*}=\mathbf{m}_{\mathbf{e}}-\frac{\varepsilon^{2}}{4} \int_{\Omega}\left|\nabla u^{\mathbf{e}}\right|^{4}+o\left(\varepsilon^{2}\right) .
$$

It is clear that (up to multiplication with an $\alpha \in \mathbb{S}^{1}$ ) we have $u^{\mathbf{d}}=v^{d}$ and $u^{\mathbf{e}}=v^{d-1}$, where $v=u^{(1,1)}$. Thus

$$
\mathbf{m}_{\mathbf{e}, \varepsilon}=\mathbf{m}_{\mathbf{e}}-\frac{(d-1)^{4} \varepsilon^{2}}{4} \int_{\Omega}|\nabla v|^{4}+o\left(\varepsilon^{2}\right) .
$$

\footnotetext{
${ }^{14}$ This is an easy consequence of Proposition 4.2.
} 
On the other hand, we have

$$
\mathbf{m}_{\mathbf{d}, \varepsilon} \leq \mathbf{m}_{\mathbf{d}, \varepsilon}^{*}=\mathbf{m}_{\mathbf{d}}-\frac{d^{4} \varepsilon^{2}}{4} \int_{\Omega}|\nabla v|^{4}+o\left(\varepsilon^{2}\right) .
$$

If we combine (8.2) and (8.3) with (8.4) and (8.5) and with Conjecture 2, we obtain the contradiction $\mathbf{m}_{\mathbf{d}}-\frac{d^{4} \varepsilon^{2}}{4} \int_{\Omega}|\nabla v|^{4} \geq \mathbf{m}_{\mathbf{d}}-\frac{(d-1)^{4} \varepsilon^{2}}{4} \int_{\Omega}|\nabla v|^{4}+o\left(\varepsilon^{2}\right)$.

8.2. Remark. Proposition 8.1 still holds in multiply connected domains when $\mathbf{d}$ is of the form $\mathbf{d}=(d, d, 0, \ldots, 0)$.

Proposition 8.1 suggests that, for critical domains, a secondary criticality occurs, and that existence of minimizers is governed by the sign of the quantity

$$
\min \left\{\int_{\Omega}\left|\nabla u^{\mathbf{d}}\right|^{4}-\int_{\Omega}\left|\nabla u^{\mathbf{e}}\right|^{4} ; \mathbf{e} \in \mathscr{B}^{*}\right\} .
$$

Note that, unlike the size, this quantity is not conformally invariant. This suggests that existence of minimizers for small $\varepsilon$ may not be a conformally invariant property of the domains.

We next turn to existence of critical points in simply connected domains. Our main result in Section 6, Theorem 6.1, asserts existence of critical points of degree 1 provided $\varepsilon$ is large. Even for large $\varepsilon$, we do not know what happens in degree $\geq 2$. In an opposite direction, in a work in progress [27] we investigate with Lamy existence of critical points for small $\varepsilon$. Our starting point is the observation that, when $\Omega=\mathbb{D}$, we may explicitly construct radial critical points of $E_{\varepsilon}$ in $\mathscr{E}_{d}$. This is done by the minimization of $E_{\varepsilon}$ in the class of the maps of the form $f(r) e^{d \imath \theta}$. Using inverse function theorem methods in the spirit of Pacard and Rivière [30], we obtain persistence of critical points when $d=1$ and $\Omega$ is close to the unit disc. More precisely, we establish the following

8.3. Theorem. There exist some $\delta>0$ and $\varepsilon>0$ such that the following hold. If there exists $a$ conformal representation $f: \mathbb{D} \rightarrow \Omega$ such that $f \in C^{1, \alpha}$ and $\|f-I d\|_{C^{1, \alpha}}<\delta$, then, for $\varepsilon<\varepsilon_{0}$, there exist critical points of $E_{\varepsilon}$ with prescribed degree 1 in $\Omega$.

More generally, we derive necessary conditions in order to have existence of critical points for arbitrary $d$ and in an arbitrary simply connected domain $\Omega$. Without giving details here, these conditions ${ }^{15}$ are presumably "generically" satisfied, that is, we establish existence of critical points in "most" of domains.

The above critical points are qualitatively different form the ones obtained by Berlyand and Rybalko [11] and described in Section 5. Indeed, the radial critical points vanish at the origin and have modulus close to 1 outside a small neighborhood of the origin. On the other hand, the critical points constructed in [11] have uniformly bounded energy and thus have modulus close to 1 outside a thin boundary layer. The former critical points have inner vortices; the latter, boundary vortices. In contrast with the case of the disc, it is not known whether critical points with inner vortices exist in multiply connected domains. However, it is quite likely that the analysis in [28] extends to multiply connected domains, and that such critical points do indeed "generically" exist.

\section{REFERENCES}

[1] L. Almeida. Topological sectors for Ginzburg-Landau energies. Rev. Mat. Iberoamericana, 15(3):487-545, 1999.

[2] A. Ambrosetti and P.H. Rabinowitz. Dual variational methods in critical point theory and applications. J. Funct. Anal., 14:349-381, 1973.

[3] T. Aubin. Équations différentielles non linéaires et problème de Yamabe concernant la courbure scalaire. J. Math. Pures Appl. (9), 55(3):269-296, 1976.

[4] A. Bahri and J.-M. Coron. On a nonlinear elliptic equation involving the critical Sobolev exponent: the effect of the topology of the domain. Comm. Pure Appl. Math., 41(3):253-294, 1988.

[5] L.V. Berlyand, D. Golovaty, and V. Rybalko. Nonexistence of Ginzburg-Landau minimizers with prescribed degree on the boundary of a doubly connected domain. C. R. Math. Acad. Sci. Paris, 343(1):63-68, 2006.

\footnotetext{
${ }^{15}$ The first condition requires that a certain smooth function, which depends on $\Omega$ and has critical points, does have a nondegenerate critical point. The second one requires that a certain Fredholm operator of zero index and depending on $\Omega$, is invertible.
} 
[6] L.V. Berlyand, D. Golovaty, and V. Rybalko. Capacity of a multiply-connected domain and nonexistence of Ginzburg-Landau minimizers with prescribed degrees on the boundary. arXiv:math/0601018v4, 2008.

[7] L.V. Berlyand and P. Mironescu. Ginzburg-Landau minimizers with prescribed degrees. Capacity of the domain and emergence of vortices. J. Funct. Anal., 239(1):76-99, 2006.

[8] L.V. Berlyand and P. Mironescu. Ginzburg-Landau minimizers in perforated domains with prescribed degrees. http://math.univ-lyon1.fr/ mironescu/3.pdf, 2008.

[9] L.V. Berlyand and P. Mironescu. Two-parameter homogenization for a Ginzburg-Landau problem in a perforated domain. Netw. Heterog. Media, 3(3):461-487, 2008.

[10] L.V. Berlyand, P. Mironescu, V. Rybalko, and E. Sandier. Minimax Critical Points in Ginzburg-Landau Problems with Semi-stiff Boundary Conditions: Existence and Bubbling. http://hal.archives-ouvertes.fr/hal-00747639, 2012.

[11] L.V. Berlyand and V. Rybalko. Solutions with vortices of a semi-stiff boundary value problem for the GinzburgLandau equation. J. Eur. Math. Soc. (JEMS), 12(6):1497-1531, 2010.

[12] L.V. Berlyand and K. Voss. Symmetry Breaking in Annular Domains for a Ginzburg-Landau Superconductivity Mode. In R.C. McPhedran, L.C. Botten, and N.A.P. Nicorovici, editors, IUTAM Symposium on Mechanical and Electromagnetic Waves in Structured Media, volume 91 of Solid Mechanics and its Applications, pages 189-200. Kluwer Academic Publishers, 2001.

[13] F. Bethuel, H. Brezis, and F. Hélein. Asymptotics for the minimization of a Ginzburg-Landau functional. Calc. Var. Partial Differential Equations, 1(2):123-148, 1993.

[14] F. Bethuel, H. Brezis, and F. Hélein. Ginzburg-Landau vortices. Progress in Nonlinear Differential Equations and their Applications, 13. Birkhäuser Boston Inc., Boston, MA, 1994.

[15] F. Bethuel and J.-M. Ghidaglia. Improved regularity of solutions to elliptic equations involving Jacobians and applications. J. Math. Pures Appl. (9), 72(5):441-474, 1993.

[16] A. Boutet de Monvel-Berthier, V. Georgescu, and R. Purice. A boundary value problem related to the GinzburgLandau model. Comm. Math. Phys., 142(1):1-23, 1991.

[17] H. Brezis. Degree theory: old and new. In Topological nonlinear analysis, II (Frascati, 1995), volume 27 of Progr. Nonlinear Differential Equations Appl., pages 87-108. Birkhäuser Boston, Boston, MA, 1997.

[18] H. Brezis and P. Mironescu. Sobolev Maps with Values into the Circle. Analytical, Geometrical and Topological Aspects. In preparation.

[19] H. Brezis and L. Nirenberg. $H^{1}$ versus $C^{1}$ local minimizers. C. R. Acad. Sci. Paris Sér. I Math., 317(5):465-472, 1993.

[20] H. Brezis and L. Nirenberg. Degree theory and BMO. I. Compact manifolds without boundaries. Selecta Math. (N.S.), 1(2):197-263, 1995.

[21] H. Brezis and L. Nirenberg. Degree Theory and BMO. II. Compact manifolds with boundaries. Selecta Math. (N.S.), 2:309-368, 1996.

[22] M. Dos Santos. Local minimizers of the Ginzburg-Landau functional with prescribed degrees. J. Funct. Anal., 257(4):1053-1091, 2009.

[23] A. Farina and P. Mironescu. Uniqueness of vortexless Ginzburg-Landau type minimizers in two dimensions. Calc. Var. PDE, to appear.

[24] D. Golovaty and L.V. Berlyand. On uniqueness of vector-valued minimizers of the Ginzburg-Landau functional in annular domains. Calc. Var. Partial Differential Equations, 14(2):213-232, 2002.

[25] F. Hélein. Constant mean curvature surfaces, harmonic maps and integrable systems. Lectures in Mathematics ETH Zürich. Birkhäuser Verlag, Basel, 2001. Notes taken by Roger Moser.

[26] A. Jaffe and C.H. Taubes. Vortices and monopoles, volume 2 of Progress in Physics. Birkhäuser Boston, Mass., 1980. Structure of static gauge theories.

[27] X. Lamy and P. Mironescu. Existence of critical points with semi-stiff boundary conditions for singular perturbation problems in simply connected planar domains. In progress.

[28] L. Lassoued and P. Mironescu. Ginzburg-Landau type energy with discontinuous constraint. J. Anal. Math., 77:1-26, 1999.

[29] P. Mironescu. Explicit bounds for solutions to a Ginzburg-Landau type equation. Rev. Roumaine Math. Pures Appl., 41(3-4):263-271, 1996.

[30] F. Pacard and T. Rivière. Linear and nonlinear aspects of vortices. Progress in Nonlinear Differential Equations and their Applications, 39. Birkhäuser Boston Inc., Boston, MA, 2000. The Ginzburg-Landau model.

[31] J. Rubinstein and P. Sternberg. Homotopy classification of minimizers of the Ginzburg-Landau energy and the existence of permanent currents. Comm. Math. Phys., 179(1):257-263, 1996.

[32] E. Sandier and S. Serfaty. Vortices in the magnetic Ginzburg-Landau model. Progress in Nonlinear Differential Equations and their Applications, 70. Birkhäuser Boston Inc., Boston, MA, 2007.

[33] S. Serfaty. Stability in 2d Ginzburg-Landau passes to the limit. Indiana Univ. Math. J., 54(1):199-221, 2005.

[34] N.S. Trudinger. Remarks concerning the conformal deformation of Riemannian structures on compact manifolds. Ann. Scuola Norm. Sup. Pisa (3), 22:265-274, 1968.

[35] K.K. Uhlenbeck. Removable singularities in Yang-Mills fields. Comm. Math. Phys., 83(1):11-29, 1982. 
UNIVERsité DE LYON, UNIVERsité LYON 1, CNRS UMR5208, INSTITUT CAMille JoRDAN, 43 BLVD DU 11 NOVEMBRE 1918, F-69622 VilleuRBANNE-CEDEX, FRANCE

E-mail address: mironescu@math.univ-lyon1.fr 